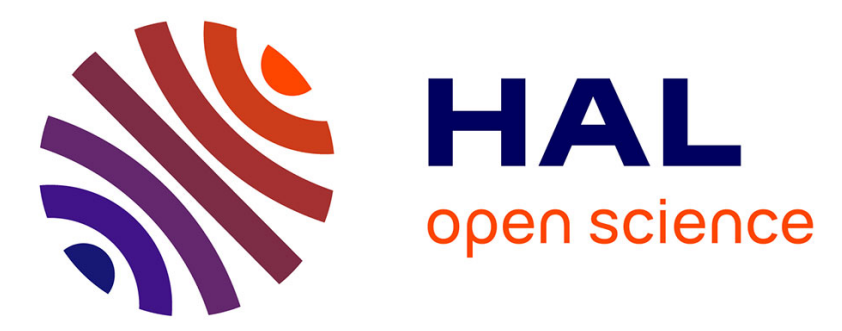

\title{
Programmable Thermoresponsive Micelle-Inspired Polymer Ionic Liquids as Molecular Shuttles for Anionic Payloads
}

Steffen Wiedmann, Manuel Luitz, Benjamin Kerscher, Jean-François Lutz, Rolf Mülhaupt

\section{To cite this version:}

Steffen Wiedmann, Manuel Luitz, Benjamin Kerscher, Jean-François Lutz, Rolf Mülhaupt. Programmable Thermoresponsive Micelle-Inspired Polymer Ionic Liquids as Molecular Shuttles for Anionic Payloads. Macromolecules, 2019, 52 (24), pp.9672 - 9681. 10.1021/acs.macromol.9b01621. hal-02404730

\section{HAL Id: hal-02404730 \\ https://hal.science/hal-02404730}

Submitted on 31 Dec 2020

HAL is a multi-disciplinary open access archive for the deposit and dissemination of scientific research documents, whether they are published or not. The documents may come from teaching and research institutions in France or abroad, or from public or private research centers.
L'archive ouverte pluridisciplinaire $\mathbf{H A L}$, est destinée au dépôt et à la diffusion de documents scientifiques de niveau recherche, publiés ou non, émanant des établissements d'enseignement et de recherche français ou étrangers, des laboratoires publics ou privés. 


\title{
Programmable thermoresponsive micelle-inspired
}

\author{
polymer ionic liquids as molecular shuttles for \\ anionic payloads
}

Steffen Wiedmann ${ }^{1,2,3}$, Manuel Luitz ${ }^{1,2, \neq}$, Benjamin Kerscher ${ }^{1,2}$, Jean-François Lutz ${ }^{4}$, Rolf Mülhaupt*,1,2,3

${ }^{1}$ Institute for Macromolecular Chemistry, University of Freiburg, Stefan-Meier-Str. 31, D-79104 Freiburg, Germany. E-mail: rolfmuelhaupt@web.de

${ }^{2}$ Freiburg Materials Research Center (FMF), University of Freiburg, Stefan-Meier-Str. 21, D79104 Freiburg, Germany

${ }^{3}$ Freiburg Centre for Interactive Materials and Bioinspired Technologies (FIT), University of Freiburg, Georges-Köhler-Allee 105, D-79110 Freiburg, Germany

${ }^{4}$ Institut Charles Sadron, CNRS, Université de Strasbourg, UPR22, 23 rue du Loess, 67034 Strasbourg Cedex 2, France

Present address: ${ }^{\ddagger}$ Department of Microsystems Engineering (IMTEK), Laboratory of Process Technology, University of Freiburg, Georges-Köhler-Allee 103, 79110 Freiburg im Breisgau, Germany

Published in Macromolecules, 52, 9672-9681 (2019)

https://doi.org/10.1021/acs.macromol.9b01621 
ABSTRACT. To enable the programmable thermoresponsive transport of anions between two phases, multifunctional hyperbranched polymer ionic liquids (hyperPILs) exhibiting micelleinspired architectures are tailored as molecular shuttles. These polyelectrolytes consist of a hyperbranched poly(3-ethyl-3-hydroxymethyloxetane) (PEHO) core, an inner polyionic imidazolium $\left(\mathrm{Im}^{+}\right)$and an outer thermoresponsive polyoxazoline (POx) shell, which exhibits lower critical solution temperature (LCST) behavior in aqueous medium. Key step of the hyperPIL synthesis is the efficient chain termination of the cationic ring-opening 2-oxazoline polymerization by addition of polyfunctional imidazole-terminated PEHO. The resulting covalent attachment of the LCST-polyoxazoline shell renders hyperPIL polyelectrolytes thermoresponsive. As a function of the polyoxazoline chain length and the oxazoline monomer type, the hyperPIL cloud points $\left(T_{\mathrm{CP}}\right)$ vary over a wide temperature range. The inner imidazolium shell enables the immobilization and transport of various anionically charged organic and inorganic payloads via anion exchange. Due to the thermal switching of the hydrophilicity/hydrophobicity balance, hyperPILs function as programmable molecular shuttles for anionic payloads transported back and forth between the phases of ethyl acetate and water. Thus, switchable hyperPILs qualify for manifold potential applications like catalytic processes with facile recycling of homogeneous catalysts via phase transfer. 


\section{INTRODUCTION}

Micelles and micellar nanostructures play an outstanding role in biological and industrial processes. ${ }^{1-5}$ Therefore, the exploration of micelle-inspired polymer structures represents a fascinating field in polymer chemistry, since they can be used as functional nanocompartments and transporters. Typically, such micellar polymer systems are based on self-assembly of amphiphilic block copolymers. However, many of the resulting supramolecular assemblies are highly sensitive to shear and changes of $\mathrm{pH}$ or temperature, which frequently account for disintegration of the micelles accompanied by loss of their function. This risk is avoided by creating robust nanostructured core/shell polymers resembling unimolecular micelles. ${ }^{6}$

Polymers containing either repeat units or functional groups similar to ionic liquids (ILs) often referred to as polymer ionic liquids (PILs) - have established themselves in the group of polyelectrolytes as an intriguing subclass of materials exhibiting exceptional characteristics. ${ }^{7}$ Recently, owing to their unique combination of properties and related expected applications, the number of studies on thermoresponsive PILs, in particular ones with lower critical solution temperature (LCST) behavior in water, has increased rapidly. ${ }^{7}$ One strategy towards thermoresponsive PILs is homopolymerization of already thermoresponsive IL monomers. This strategy, pioneered by the groups of Ohno and Yuan, ${ }^{8,9}$ was exploited for the synthesis of a variety of LCST PILs with polycationic or polyanionic backbone. ${ }^{10-13}$ The second approach is the design of random or block copolymers of an IL monomer and a non-ionic monomer with at least one of them showing thermoresponsive behavior in polymerized form. Most prominent examples are copolymers of different imidazolium-type IL monomers and $N$ isopropylacrylamide (NIPAM). ${ }^{14-19}$ Similar to thermoresponsive PILs, stimulus-responsive hyperbranched macromolecules are attracting great attention. ${ }^{20}$ In a recent advance and as a rare 
example of thermoresponsive PILs with highly branched architecture, Tsukruk et al. tailored core/shell polymers composed of hyperbranched carboxylate-functionalized polyester cores and ammonium-terminated poly( $N$-isopropylacrylamide) (PNIPAM) chains bonded to the core molecules as thermoresponsive corona by ionic interactions. ${ }^{21}$

In the past, hyperbranched polymer ionic liquids (hyperPILs) with onion-like compartmentalized topology have been developed in our group, which combine the features of PIL materials with the benefits of unimolecular micellar architecture. ${ }^{22}$ They are composed of a hyperbranched poly(3-ethyl-3-hydroxymethyloxetane) (PEHO) core, an inner ionic shell of covalently attached imidazolium cations with tosylate counter anions, and a non-polar outer shell of alkyl chains. These micelle-inspired polyelectrolytes function as highly effective molecular transporters in two-phase systems and enable phase transfer of molecules and nanomaterials from water into immiscible non-polar organic solvents. ${ }^{23}$ However, they only allow for the unidirectional and irreversible transport and do not enable shuttling of payloads back and forth between two phases. The programmable thermal switching of the transport between two phases is highly desirable for many applications including catalyst recycling. Hence, aiming at making this phase transfer reversible and switchable we have developed stimulus-responsive micellar core/shell hyperPILs as molecular shuttles. This is achieved by equipping the hyperPILs with an outer shell of thermoresponsive polyoxazoline segments exhibiting LCST behavior in aqueous media. Whereas this outer LCST-polyoxazoline shell enables thermal switching of the hydrophilicity/hydrophobicity balance, the cationic imidazolium coupling groups as inner shell account for the immobilization of anionic payloads via anion exchange.

During recent years, non-ionic thermoresponsive polymer systems have been tailored as molecular shuttles for the reversible transport of payloads back and forth between two liquid 
phases in a controlled manner. Most of them exploit self-assembly and the formation of block copolymer micelles. For example, since 2006, the Lodge group has pioneered such systems based on micelle-forming poly((1,2-butadien)-b-ethylene oxide), poly( $N$-isopropylacrylamide- $b$ ethylene oxide), and poly(ethylene oxide- $b$-propylene oxide- $b$-ethylene oxide) block copolymers, which can reversibly transport dye molecules as payloads back and forth between water and a water-immiscible IL. This shuttling process is controlled by temperature changes. ${ }^{24-28}$ Schubert's group demonstrated that also polyoxazoline block copolymers function as thermoreversibly switchable transporters in the two-phase systems used by Lodge. ${ }^{29,30}$ Apart from block copolymers, appropriately designed polymer brush-grafted "hairy particles" were shown to reversibly travel back and forth between two phases not only in water/IL systems, ${ }^{31,32}$ but also in two-phase systems consisting of water and an organic solvent. ${ }^{33,34}$ For example, in octane/water, thermoresponsive hairy particles, like poly(methoxytri(ethylene glycol) methacrylate) grafted on a cross-linked poly(acrylic acid) core, were used as carriers for palladium nanoparticles, which catalyzed hydrogenation reactions and were easily recycled. ${ }^{35}$ However, to the best of our knowledge, the use of polyionic micelle-inspired macromolecules as thermoresponsive shuttles in two-phase systems has not been reported to date.

The novel hyperPILs presented herein are readily obtained by termination of the cationic ringopening 2-oxazoline polymerization with polyfunctional imidazole-terminated hyperbranched PEHO to covalently attach LCST-polyoxazolines to the PEHO core via cationic imidazolium groups as IL moieties. By the facile variations of the size and composition of the polyoxazoline segments, the cloud point $\left(T_{\mathrm{CP}}\right)$ of the core/shell polyelectrolytes in aqueous solution is readily adjusted. Special attention is paid to temperature-induced transport in two-phase water/organic solvent systems using various payloads to unveil the transport mechanism and to demonstrate the 
concept of switchable hyperPILs as thermoresponsive molecular shuttles operating in two-phase systems.

\section{RESULTS AND DISCUSSION}

The synthetic route for the preparation of the thermoresponsive hyperPILs with micelleinspired core/shell architectures is shown in Scheme 1. The hyperPIL unimolecular micelles are readily obtained via a "grafting-to" process in which the cationic ring-opening polymerization of 2-oxazolines is terminated with polyfunctional imidazole-terminated hyperbranched poly(3ethyl-3-hydroxymethyloxetane) (PEHO-Im), which is prepared in a three step synthesis following previously reported procedures. ${ }^{22,36}$ This chain termination reaction affords coupling of PEHO-Im and poly(2-oxazoline)s via the nucleophilic attack of the imidazole groups at the terminal oxazolinium rings to produce the inner shell consisting of imidazolium cations with tosylate counter anions. This strategy of using imidazole-functionalized polymers as macroterminating agents for living polyoxazoline chains is very versatile and has been presented as an efficient method for the synthesis of ionic polyisobutylene-b-poly(2-ethyl-2-oxazoline) diblock copolymers. ${ }^{37}$ 
Scheme 1: Synthesis and molecular architectures of thermoresponsive hyperPILs.

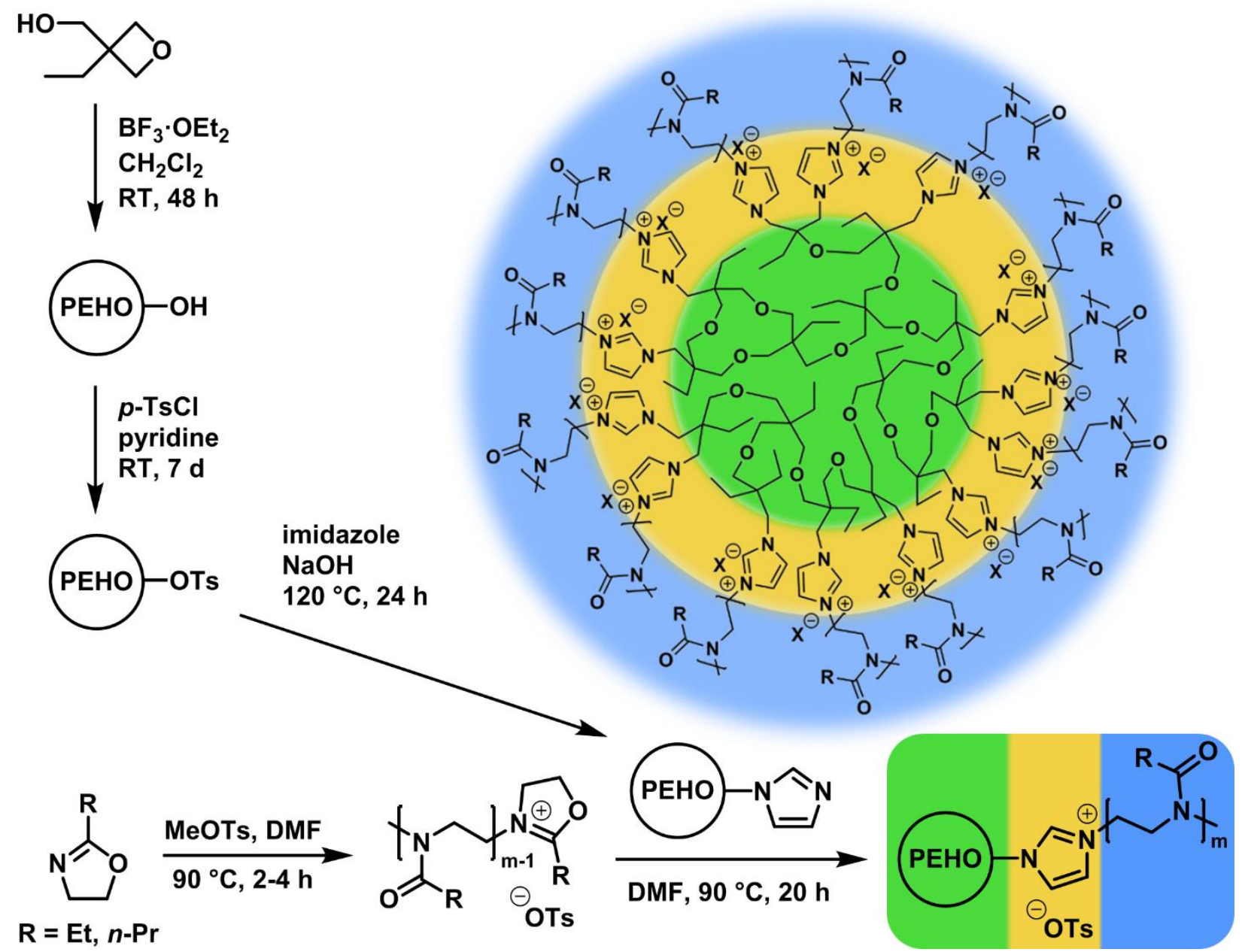


Synthesis of the hyperPILs. The synthesis of PEHO-OH was performed by cationic ringopening polymerization of 3-ethyl-3-hydroxymethyloxetane $(\mathrm{EHO})$ using $\mathrm{BF}_{3} \cdot \mathrm{OEt}_{2}$ as initiator, following a published procedure. ${ }^{38}$ The obtained hyperbranched PEHO-OH was identified by ${ }^{1} \mathrm{H}$ and ${ }^{13} \mathrm{C}$ NMR spectroscopy (for Figures of NMR spectra, SEC, TGA, and DSC curves of PEHO-OH and its derivatives see SI). Direct investigation of the molecular weight distribution using size exclusion chromatography (SEC) failed due to its poor solubility in the solvents commonly used for this purpose. The degree of branching of the PEHO-OH is $46 \%$ as determined by ${ }^{13} \mathrm{C}$ inverse-gated NMR spectroscopy of the trifluoroacetylated derivative (PEHOTFA).

In order to enable the substitution of the hydroxy groups by imidazole, the PEHO-OH was subsequently treated with tosyl chloride to form the tosylate derivative PEHO-OTs, as previously described by our group. ${ }^{22}$ According to ${ }^{1} \mathrm{H}$ NMR spectroscopy, a high degree of functionalization (DF) of $90 \%$ was achieved. SEC analysis revealed a number-average molecular weight $\left(M_{\mathrm{n}}\right)$ of $3800 \mathrm{~g} \mathrm{~mol}^{-1}$ and, as a result of chain transfer occurring as side reaction during EHO polymerization, ${ }^{39}$ a dispersity $(\nexists)$ of 2.0 . However, since calibration of SEC with linear standards usually underestimates the molecular weight as well as the disperisty of hyperbranched polymers, these numbers have to be considered with appropriate care. From $M_{\mathrm{n}}$ of PEHO-OTs, the degree of polymerization (DP) of the PEHO core was calculated to be 15 , which corresponds to a molar mass of the original PEHO-OH of $1700 \mathrm{~g} \mathrm{~mol}^{-1}$. Finally, the tosyl groups were substituted by imidazole, ${ }^{36}$ thereby obtaining PEHO-Im with a high DF of $82 \%$. SEC measurement failed due to interactions of the imidazole groups with the stationary phase. However, based on DP of PEHO-OTs and taking into account DF, $M_{\mathrm{n}}$ of PEHO-Im was calculated to be $2500 \mathrm{~g} \mathrm{~mol}^{-1}$. 
The yields of the polymerization and the polymer-analogous reactions were very high and amounted to at least $90 \%$ each, which resulted in a total yield of remarkable $78 \%$ over all the three steps. As expected, thermal analysis showed that all PEHO derivatives were amorphous substances with strongly end group-dependent glass transition $\left(T_{\mathrm{g}}\right)$ and onset decomposition temperatures $\left(T_{\mathrm{O}}\right)$. Generally, the $T_{\mathrm{g}}$ values of hyperbranched macromolecules increase with higher end group polarity, i.e. stronger end group interaction, which is reflected in the higher values for PEHO-OH $\left(40^{\circ} \mathrm{C}\right)$ and PEHO-Im $\left(52{ }^{\circ} \mathrm{C}\right)$ compared to PEHO-OTs $\left(23^{\circ} \mathrm{C}\right)$. It is worth to mention the extraordinary thermal stability of the PEHO-Im, which does not show significant mass loss up to temperatures of almost $400{ }^{\circ} \mathrm{C}$, even under air. The substantially lower decomposition temperature of PEHO-OTs is explained, in analogy to the structurally related neopentyltosylate, ${ }^{40}$ by rearrangement of the end groups followed by elimination of $p$-toluene sulfonic acid. The most important characteristics of $\mathrm{PEHO}-\mathrm{OH}$ and its derivatives are summarized in Table 1.

Table 1: Properties of PEHO-OH and its derivatives.

\begin{tabular}{lcccccc}
\hline Sample code & $\begin{array}{c}\mathrm{DF}^{\mathrm{a}} \\
{[\%]}\end{array}$ & $\begin{array}{c}M_{\mathrm{n}} \\
{\left[\mathrm{g} \mathrm{mol}^{-1}\right]}\end{array}$ & $\begin{array}{c}\text { Yield } \\
{[\%]}\end{array}$ & $\begin{array}{c}T_{\mathrm{g}}^{\mathrm{d}} \\
{\left[{ }^{\circ} \mathrm{C}\right]}\end{array}$ & $\begin{array}{c}T_{\mathrm{O}}^{\mathrm{e}}(\mathrm{air}) \\
{\left[{ }^{\circ} \mathrm{C}\right]}\end{array}$ & $\begin{array}{c}T_{\mathrm{O}}{ }^{\mathrm{e}}\left(\mathrm{N}_{2}\right) \\
{\left[{ }^{\circ} \mathrm{C}\right]}\end{array}$ \\
\hline PEHO-OH & 100 & $1700^{\mathrm{c}}$ & 90 & 40 & 340 & 401 \\
PEHO-OTs & 90 & $3800^{\mathrm{b}}$ & 91 & 23 & 235 & 299 \\
PEHO-Im & 82 & $2500^{\mathrm{c}}$ & 95 & 52 & 402 & 404
\end{tabular}

\footnotetext{
${ }^{a}$ End group fraction of the name-giving functional groups, according to ${ }^{1} \mathrm{H}$ NMR spectroscopy; ${ }^{b}$ determined by SEC $\left(\bigoplus=2.0\right.$; calculated from $M_{\mathrm{n}}$ : DP $\left.=15\right)$; ${ }^{\mathrm{c}}$ calculated from DP and average molar mass of the respective linear repeat unit (for further explanations see SI); determined by DSC; 'determined by TGA.

To synthesize the thermoresponsive hyperPILs, poly(2-alkyl-2-oxazoline)s were grafted onto PEHO-Im core molecules in a "grafting-to" process. The oxazoline polymerization was
} 
performed by means of a cationic ring-opening process with methyl tosylate (MeOTs) as initiator. After complete consumption of the monomer, PEHO-Im was added to the solution as terminating agent of the cationic oxazoline polymerization in which most chains contained cationic oxazolinium rings as end groups. Dimethylformamide (DMF) was chosen as solvent, which, due to its polar aprotic nature, enables both the cationic ring-opening oxazoline polymerization and the dissolution of PEHO-Im. The monomers used were 2-ethyl-2-oxazoline (EtOx), 2-n-propyl-2-oxazoline ( $n \mathrm{PrOx}$ ), and a 1:1 molar mixture of the two monomers (EtOx $/ n$ PrOx) producing random copolymers. In addition, the DP of the polyoxazoline segments was varied by the molar ratio of monomer to initiator (10:1 or 20:1). A ratio of 1.1:1 was chosen as the molar ratio of initiator to imidazole groups of the PEHO-Im to achieve complete functionalization of the imidazole groups. Six hyperPILs with different polyoxazoline shells were synthesized, which are abbreviated as PEHO-IL-POx $(\mathrm{m}=$ theoretical DP of the polyoxazoline shell according to monomer:initiator ratio).

The thermoresponsive hyperPILs were isolated by precipitation of the reaction mixture in $\mathrm{Et}_{2} \mathrm{O}$, which in the case of the two PEHO-IL-P $n$ PrOx samples and PEHO-IL-P(EtOx/ $n$ PrOx $)_{10}$ afforded core/shell polymers with acceptable purity (max. 4 wt.\% low-molecular-weight components, which correspond to the fraction below $5 \mathrm{~kg} \mathrm{~mol}^{-1}$ for $\mathrm{m}=10$ and below $10 \mathrm{~kg} \mathrm{~mol}^{-1}$ for $\mathrm{m}=20$ in the SEC curves; see Table S2 $+\mathrm{S} 3$ and Figure S14). For the other samples, no satisfactory purity was achieved in this process due to poor solubility of the respective free polyoxazolines in $\mathrm{Et}_{2} \mathrm{O}$. To separate the oxazoline homopolymer byproducts, the two PEHO-IL-PEtOx samples and PEHO-IL-P(EtOx $/ n$ PrOx $)_{20}$ were therefore subjected to a further purification step. For this purpose, highly concentrated aqueous solutions $\left(\mathrm{c}=100 \mathrm{~g} \mathrm{~L}^{-1}\right)$ of the polymers were heated a few degrees above their $T_{\mathrm{CP}}$, which led to a macroscopic 
temperature-induced phase separation (TIPS). At temperatures only slightly above $T_{\mathrm{CP}}$ of the respective hyperPIL, the free polyoxazoline chains were still well soluble in water and, thus, remained in the water-rich upper phase, while the purified hyperPILs were isolated from the polymer-rich bottom phase. In this way, the amount of non-attached polyoxazoline chains was significantly reduced, as indicated by the decrease of the low-molecular-weight fraction to maximally 7 wt.\%. However, the TIPS purification process was accompanied by a massive loss of product, resulting in moderate total yields of the respective PEHO-IL-POx samples of only $24-56 \%$, whereas the PEHO-IL-PnPrOx samples and PEHO-IL-P(EtOx/nPrOx $)_{10}$ were obtained in much higher yields ranging between 74 and $85 \%$ (see Table 2).

Table 2: Properties of the hyperPILs.

\begin{tabular}{|c|c|c|c|c|c|c|}
\hline \multirow{2}{*}{ Sample code } & \multicolumn{2}{|c|}{ Polyoxazoline chain length } & \multicolumn{2}{|c|}{$M_{\mathrm{n}}\left[\mathrm{g} \mathrm{mol}^{-1}\right]$} & \multirow{2}{*}{$\begin{array}{c}Ð \\
\text { SEC }^{\mathrm{a}}\end{array}$} & \multirow{2}{*}{$\begin{array}{l}\text { Yield } \\
{[\%]}\end{array}$} \\
\hline & {$[\mathrm{M}]_{0} /[\mathrm{I}]_{0}$} & ${ }^{1} \mathrm{H}$ NMR & ${ }^{1} \mathrm{H}$ NMR & $\mathrm{SEC}^{\mathrm{a}}$ & & \\
\hline PEHO-IL-PEtOx 10 & 10 & 10.6 & 18000 & 19000 & 1.7 & 56 \\
\hline PEHO-IL-PEtOx 20 & 20 & 22.8 & 33000 & 34000 & 2.0 & 24 \\
\hline PEHO-IL-P(EtOx $/ n \operatorname{PrOx})_{10}$ & 10 & 10.8 & 19000 & 20000 & 1.6 & 85 \\
\hline PEHO-IL-P(EtOx $/ n \operatorname{PrOx})_{20}$ & 20 & 26.5 & 40000 & 37000 & 1.7 & 36 \\
\hline PEHO-IL-P $n \operatorname{PrOx}_{10}$ & 10 & 11.4 & 21000 & 22000 & 1.6 & 74 \\
\hline PEHO-IL-P $n \mathrm{PrOx}_{20}$ & 20 & 22.1 & 36000 & 32000 & 1.6 & 80 \\
\hline
\end{tabular}

aTo determine $M_{\mathrm{n}}$ and $Ð$ of the hyperPILs from the SEC curves, the corresponding highmolecular signal was used without taking into account any low-molecular-weight impurities.

The ${ }^{1} \mathrm{H}$ NMR analysis of the hyperPILs (Figure 1 exemplarily shows the ${ }^{1} \mathrm{H}$ NMR spectrum of PEHO-IL-PnPrOx 10 , for ${ }^{1} \mathrm{H}$ NMR spectra of the other hyperPILs see SI) revealed almost quantitative functionalization of the imidazole groups, as reflected by disappearance of the 
imidazole signals. Only the NMR spectrum of PEHO-IL-P(EtOx/nPrOx) $)_{20}$ showed clearly recognizable signals of unreacted imidazole groups, but reliable quantification was prevented by overlapping of the signals. By comparing the signal intensities of the polyoxazoline repeat unit directly bound to the imidazolium cation with the remaining polyoxazoline backbone, the DP of the attached polyoxazoline chains was determined. In accordance with the SEC analysis, the remaining traces of free polyoxazoline chains were neglected in this calculation. The chain lengths calculated in this way are always slightly larger than the monomer:initiator ratio, but the deviation was usually less than $15 \%$. Only the sample PEHO-IL-P(EtOx/nPrOx $)_{20}$ showed a higher deviation of $33 \%$.

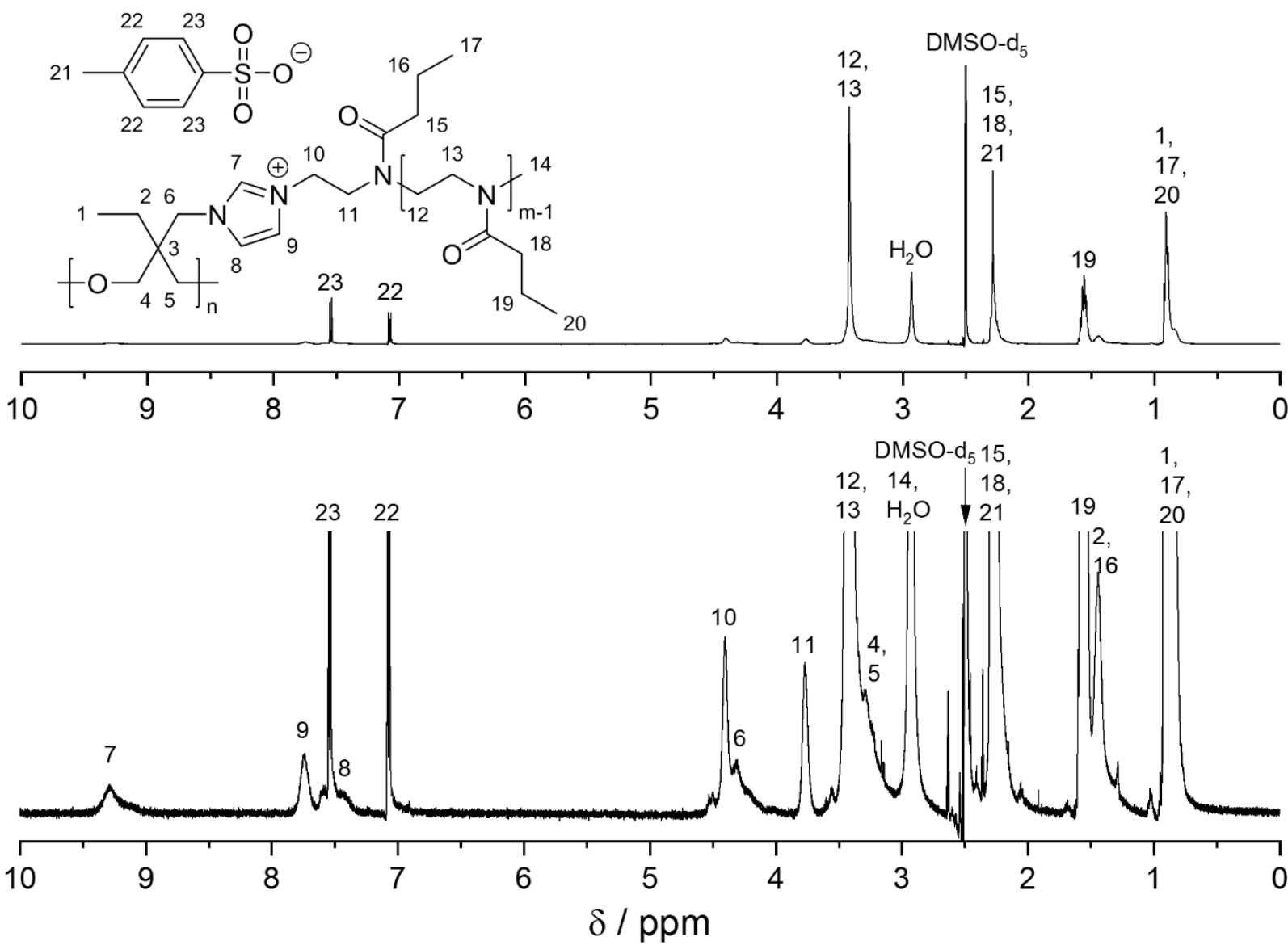

Figure 1: ${ }^{1} \mathrm{H}$ NMR spectrum of PEHO-IL-P $n \operatorname{PrOx}_{10}$ (top) and magnification of the spectrum (bottom). 
The $M_{\mathrm{n}}$ values of the hyperPILs calculated from the ${ }^{1} \mathrm{H}$ NMR spectra varied between 18000 and $21000 \mathrm{~g} \mathrm{~mol}^{-1}$ for the PEHO-IL-POx 10 samples and between 33000 and $40000 \mathrm{~g} \mathrm{~mol}^{-1}$ for the PEHO-IL-POx 20 samples. Of course, as SEC results of the PEHO-OTs intermediate are included in these calculations, these molar masses are afflicted with some uncertainty. However, even though SEC analysis of the hyperPILs is similarly problematic due to the hyperbranched core/shell architecture, the $M_{\mathrm{n}}$ values measured by SEC matched very well those ones determined by ${ }^{1} \mathrm{H}$ NMR spectroscopy and, depending on the polyoxazoline shell, fell in the range of $19000 \mathrm{~g} \mathrm{~mol}^{-1}$ (PEHO-IL-PEtOx 10$)$ to $37000 \mathrm{~g} \mathrm{~mol}^{-1}$ (PEHO-IL-P(EtOx/nPrOx) 20$)$. Thus, these numbers at least illustrate the magnitude of the molar masses of the hyperPILs. The dispersities varied from 1.6 to 2.0, however, due to the calibration with linear PMMA standards, SEC tends to underestimate these values. Table 2 summarizes the polyoxazoline chain lengths and the $M_{\mathrm{n}}$ and $Ð$ values of the hyperPILs.

The thermal analysis of the hyperPILs by DSC revealed $T_{\mathrm{g}}$ values varying between 24 and $45^{\circ} \mathrm{C}$. As expected due to the longer alkyl side-chains in $n \mathrm{PrOx},{ }^{41,42}$ the PEHO-IL-P $n$ PrOx samples had slightly lower $T_{\mathrm{g}}$ values as compared to the PEHO-IL-PEtOx or PEHO-IL$\mathrm{P}(\mathrm{EtOx} / n \mathrm{PrOx})$ samples (see Table 3$)$. The $T_{\mathrm{O}}$ values determined by TGA under air ranged between 340 and $360^{\circ} \mathrm{C}$, whereas under nitrogen atmosphere slightly higher $T_{\mathrm{O}}$ values between 350 and $370{ }^{\circ} \mathrm{C}$ were detected. In general, the differences in the decomposition temperatures of the hyperPILs are very small and a rational relation to their structure is not evident. Under air, no mass loss was detectable up to at least $200{ }^{\circ} \mathrm{C}$, under nitrogen even up to at least $300{ }^{\circ} \mathrm{C}$. Hence, the novel thermoresponsive hyperPILs exhibited the same high thermal stability as their alkylmodified predecessors. ${ }^{22}$ 
Table 3: Thermal properties of the hyperPILs.

\begin{tabular}{lccc}
\hline Sample code & $T_{\mathrm{g}}^{\mathrm{a}}\left[{ }^{\circ} \mathrm{C}\right]$ & $T_{\mathrm{O}}^{\mathrm{b}}(\mathrm{air})\left[{ }^{\circ} \mathrm{C}\right]$ & $T_{\mathrm{O}}{ }^{\mathrm{b}}\left(\mathrm{N}_{2}\right)\left[{ }^{\circ} \mathrm{C}\right]$ \\
\hline PEHO-IL-PEtOx $_{10}$ & 44 & 349 & 353 \\
PEHO-IL-PEtOx $_{20}$ & 31 & 356 & 367 \\
PEHO-IL-P(EtOx $/ n$ PrOx $)_{10}$ & 32 & 346 & 355 \\
PEHO-IL-P(EtOx $/ n$ PrOx $)_{20}$ & 45 & 358 & 365 \\
PEHO-IL-PnPrOx 10 & 24 & 339 & 353 \\
PEHO-IL-P $n P_{20}$ & 25 & 359 & 366 \\
\hline
\end{tabular}

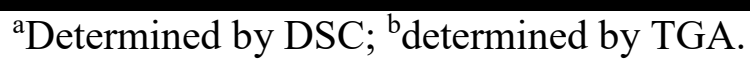

Thermoresponsive behavior of the hyperPILs. Special attention was payed to the investigation of the thermoresponsive properties of the micellar polyelectrolytes in aqueous solution. Due to the covalent attachment of the LCST-polyoxazoline shell, all six hyperPIL polyelectrolytes exhibited LCST behavior when exceeding sufficiently high concentrations. Upon heating above a concentration-dependent and material-specific temperature, clouding of the solution was observed. By subsequent cooling, the solution cleared again. This behavior, which is typical for LCST-polyoxazolines, can be explained by phase separation into a polymerrich and a water-rich phase at higher temperatures. Below $T_{\mathrm{CP}}$ the polyoxazoline chains are well dissolved due to the hydrogen bond formation between water and the polymer. Above $T_{\mathrm{CP}}$ intraand intermolecular hydrogen bonds between polyoxazoline chains are favored. ${ }^{43}$ This results in aggregation of the hyperPILs and produces polymer droplets of sufficient size to scatter light, resulting in the observed turbidity (see Scheme 2). 
Scheme 2: Clouding/clearing of an aqueous PEHO-IL-P(EtOx $/ n \operatorname{PrOx})_{20}$ solution by heating/cooling and schematic illustration of the aggregation of the hyperPILs leading to phase separation.
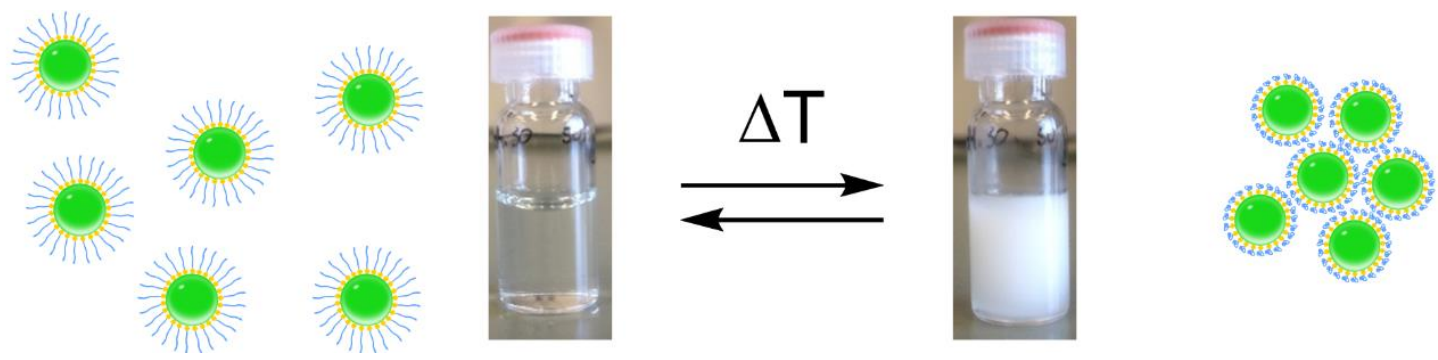

For a more detailed investigation of the thermoresponsive phase transitions and determination of $T_{\mathrm{CP}}$, temperature-dependent photometric transmission measurements were performed at different concentrations. As an example, Figure 2 shows the transmission-temperature curves of PEHO-IL-P $n \operatorname{PrOx}_{10}$ at concentrations of 5,15 , and $25 \mathrm{~g} \mathrm{~L}^{-1}$ (see Figure $\mathrm{S} 17$ for the other hyperPILs). It can easily be seen that with increasing concentration the clouding occurs at lower temperatures and the temperature range of the phase transition becomes narrower.

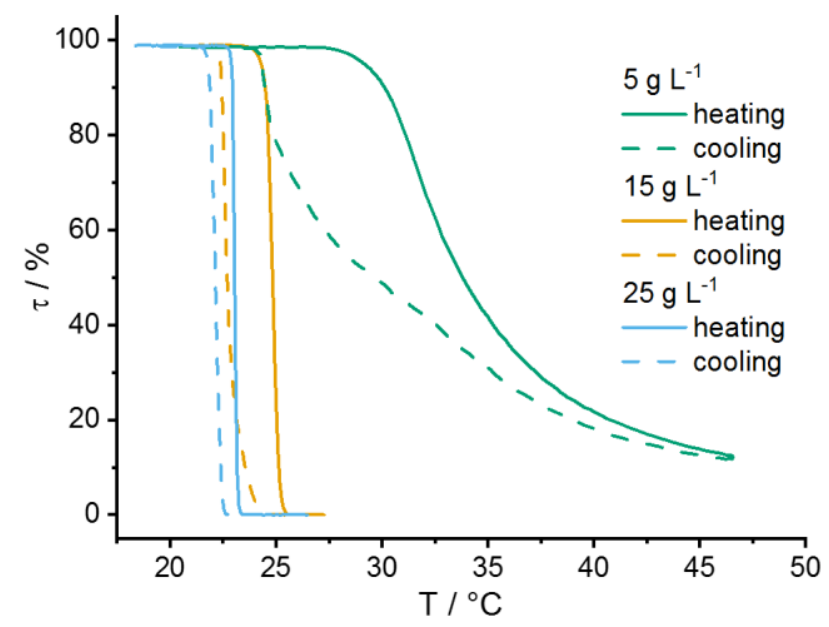

Figure 2: Transmission-temperature curves of aqueous solutions of PEHO-IL-P $n$ PrOx 10 .

The $T_{\mathrm{CP}}$ of all hyperPILs at the investigated concentrations are graphically displayed in Figure 3 (the $T_{\mathrm{CP}}$ values are listed in Table S3). The hyperPILs equipped with a PEtOx shell (PEHO-IL- 
PEtOx $_{10}$ and PEHO-IL-PEtOx 20 ) showed thermoresponsive behavior only at relatively high concentrations of $75 \mathrm{~g} \mathrm{~L}^{-1}$ and $25 \mathrm{~g} \mathrm{~L}^{-1}$, respectively, whereas for the other hyperPILs, phase transitions were detected already at much lower concentrations of $5 \mathrm{~g} \mathrm{~L}^{-1}$. Hence, the turbidimetric characterization of these PILs therefore focused on low concentrations (5$25 \mathrm{~g} \mathrm{~L}^{-1}$ ), while the PEHO-IL-PEtOx samples were examined up to a concentration of $100 \mathrm{~g} \mathrm{~L}^{-1}$.

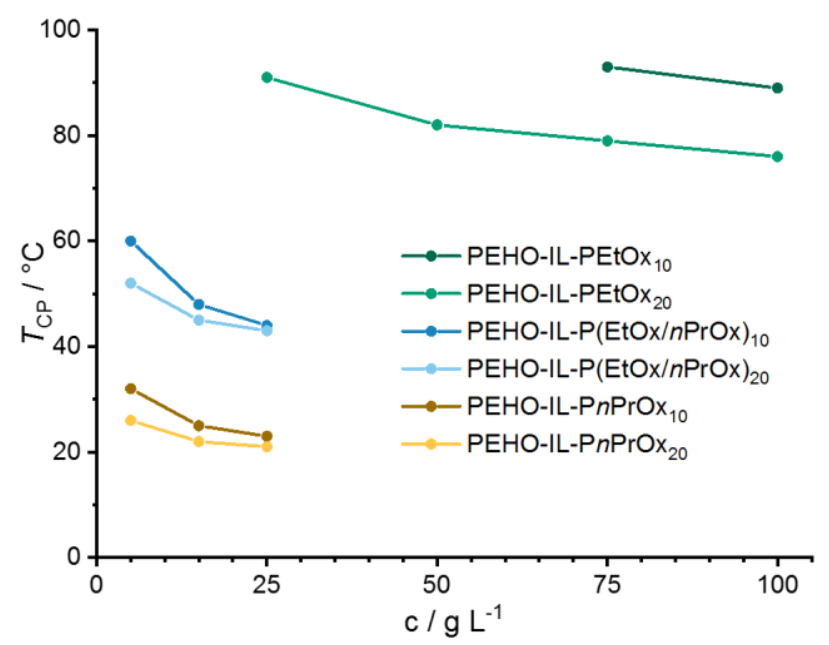

Figure 3: $T_{\mathrm{CP}}$ of aqueous hyperPIL solutions of different concentrations.

In summary, the $T_{\mathrm{CP}}$ values detected varied between 21 and $93{ }^{\circ} \mathrm{C}$. The chemical structure of the polyoxazoline shell had the strongest influence on $T_{\mathrm{CP}}$. Therefore, the values for $T_{\mathrm{CP}}$ of the PEHO-IL-PEtOx solutions $\left(76-93^{\circ} \mathrm{C}\right)$ were clearly above those of the PEHO-IL$\mathrm{P}(\mathrm{EtOx} / n \mathrm{PrOx})$ solutions $\left(43-60^{\circ} \mathrm{C}\right)$, which in turn became cloudy at considerably higher temperatures with respect to the PEHO-IL-P $n$ PrOx solutions $\left(21-32{ }^{\circ} \mathrm{C}\right)$. The second key parameter influencing the thermoresponsive behavior was the concentration. For all samples, $T_{\mathrm{CP}}$ decreased with increasing concentration. For example, $T_{\mathrm{CP}}$ of PEHO-IL-PEtOx 20 decreased by $16^{\circ} \mathrm{C}$ when increasing the concentration from 25 to $100 \mathrm{~g} \mathrm{~L}^{-1}$. As a third parameter, for the same chemical composition and concentration increasing the polyoxazoline chain length accounted for lowering $T_{\mathrm{CP}}$. Especially the $T_{\mathrm{CP}}$ values of PEHO-IL-PEtOx solutions were found to be strongly 
dependent on the size of the shell. The same trend was observed for the other hyperPILs, but the differences were less pronounced and significant changes only occurred at the lowest concentration of $5 \mathrm{~g} \mathrm{~L}^{-1}$. The effect of longer alkyl side-chain substituents on $T_{\mathrm{CP}}$ is in accord with linear polyoxazolines and can be explained by the resulting decrease of hydrophilicity. In fact, the observed influences of concentration and molar mass on $T_{\mathrm{CP}}$ were very similar to those observed for polyoxazoline homo- and copolymers. ${ }^{44}$ As a result of the turbidimetric investigations, it can be concluded that by appropriately selecting the ratio of the oxazoline monomers and the DP of the polyoxazoline chains, the thermoresponsive behavior can be readily adjusted to tailor hyperPILs with programmable $T_{\mathrm{CP}}$.

TEM. The representative TEM images of PEHO-IL-P(EtOx/ $n$ PrOx $)_{10}$ and PEHO-IL$(\mathrm{PEtOx} / n \operatorname{PrOx})_{20}$ displayed in Figure 4 show single circular particles with an average diameters with standard deviation of $20.7 \pm 4.3 \mathrm{~nm}$ and $22.4 \pm 4.8 \mathrm{~nm}$ respectively. Surprisingly, the length of the polyoxazoline segments has no substantial influence on the particle size. Therefore, the particles seem to represent aggregates of a small number of core-shell macromolecules, A single hyperPIL molecule has an average diameter not larger than 6 or $9 \mathrm{~nm}$ respectively, depending on the length of the polyoxazoline segments, as estimated by distance measurements on simulated 3D models. Samples prepared from more diluted solutions showed empty grids instead of the expected single macromolecules, probably because the small amount of deposited material was removed by the staining procedure with uranyl acetate solution. However, the size distribution of the particles is rather homogeneous, suggesting an energetic minimum at the observed diameter of about $20 \mathrm{~nm}$. Images obtained from the other hyperPILs show particles with diameters around $20 \mathrm{~nm}$ aswell, yet the contrast is too low for a solid evaluation of the average size (see Figure S19). In general, the results obtained from TEM can only give an idea about the micellar 
morphology of the dissolved hyperPILs as upon drying previously individually disolved macromolecules might fuse together to the observed larger structures. Cryo-TEM meassurements, which would give a more accurate picture of the conditions in solutions, unfortunately showed no material.
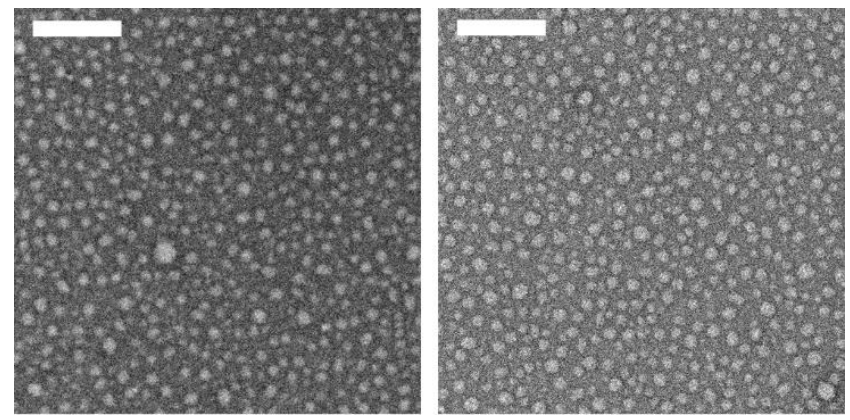

Figure 4: TEM images of PEHO-IL-P(EtOx $/ n$ PrOx) $)_{10}$ (left) and PEHO-IL-P(EtOx $\left./ n \operatorname{PrOx}\right)_{20}$ (right); scale bar $=200 \mathrm{~nm}$.

HyperPILs as thermoresponsive transporters. The thermal switching of the polarity and the hydrophilicity/hydrophobicity balance of the polyoxazoline shell accounted for a temperaturedependent phase change of the hyperPILs in two-phase systems consisting of water and an immiscible organic solvent. At temperatures below $T_{\mathrm{CP}}$, the hyperPILs were soluble in the aqueous phase, provided that water is the better solvent as compared to the organic phase. Above $T_{\mathrm{CP}}$, the hyperPILs became less polar and were rendered insoluble in water, prompting them to migrate into the organic phase. Thus, the hyperPILs can travel back and forth between aqueous and organic medium, depending on the temperature. This holds great prospects for the controlled and reversible shuttling of payloads between the two phases.

In order to investigate the temperature-induced phase change and to evaluate potential applications of the hyperPILs as programmable shuttles, the water-soluble anionic dye Congo red was used as model substance to be transported. The PEHO-IL-PEtOx samples were excluded 
from these transport experiments, since they showed thermoresponsive behavior only at relatively high concentrations and because their $T_{\mathrm{CP}}$ values were above the boiling points of many common organic solvents. Depending on the selection of the organic solvent, different observations were made. In case of relatively polar solvents such as chloroform, an immediate phase transfer of hyperPIL and dye into the organic phase occurred already at room temperature. When an aqueous Congo red/hyperPIL solution was added to chloroform, discoloration of the aqueous phase and coloration of the organic phase was observed. This state was maintained regardless of temperature. Thus, chloroform was always a better solvent for the hyperPILs than water, which prevented shuttling in this two-phase system. On the other hand, highly non-polar solvents such as $n$-hexane did not dissolve the hyperPILs, irrespective of the temperature, which impeded any phase transfer of the dye in such systems.

In the ethyl acetate/water system, however, the hyperPILs showed a temperature-dependent phase change and enabled the desired switchable shuttling of Congo red. The best results regarding reversibility and completeness of the phase transfer were achieved with PEHO-IL$\mathrm{P} n \mathrm{PrOx}_{10}$. Figure 5 illustrates this hyperPIL-mediated Congo red transport. At temperatures below the $T_{\mathrm{CP}}$ of the hyperPIL, the dye was dissolved in the aqueous phase. Upon heating to $60{ }^{\circ} \mathrm{C}$, the hyperPIL transported the dye into the organic medium. Cooling to $4{ }^{\circ} \mathrm{C}$ induced shuttling via the reversed transport of both hyperPIL and dye into the aqueous phase. The employed PEHO-IL-P $n$ PrOx $_{10}$ carrier contains about $0.6 \mathrm{mmol} \mathrm{g}^{-1}$ of cations and shuttled $0.8 \mathrm{~g}$ of Congo red anions per $1 \mathrm{~g}$ of hyperPIL. Although maximal transport capacity was not studied, this illustrates the substantial shuttling capability of the hyperPILs. This shuttling process was repeated several times without encountering noticeable differences. 


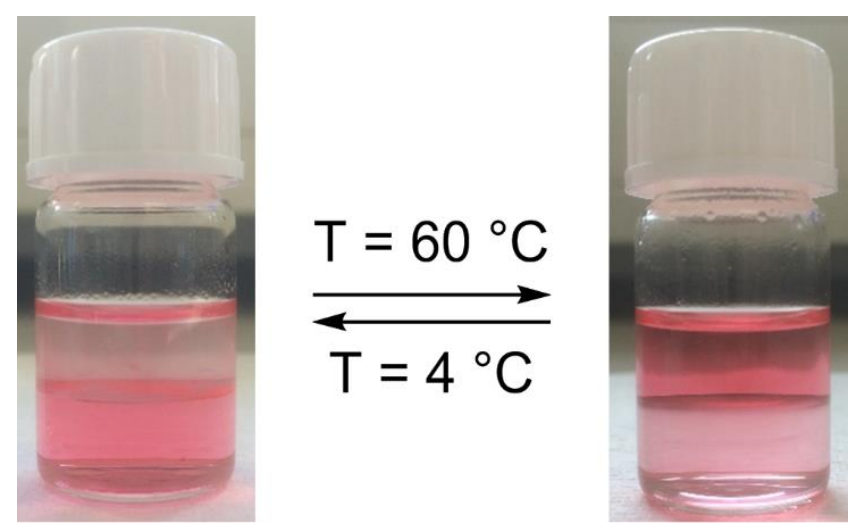

Figure 5: Temperature-controlled shuttling of Congo red in ethyl acetate/water using PEHO-IL$\mathrm{P}_{n} \mathrm{PrOx}_{10}$ as molecular shuttle.

For elucidation of the transport mechanism, identical experiments were performed with a water-soluble cationic dye (methylene blue) and a water-insoluble non-ionic dye (Sudan IV) (see Figure S18 for structures of the investigated dyes). In both cases, a temperature-induced hyperPIL-mediated shuttling could not be observed. The polar methylene blue always remained in the aqueous phase, whereas the non-polar Sudan IV always stayed in the ethyl acetate phase. In view of these results, it is apparent that shuttling only worked with anionic dye payloads as they were immobilized on hyperPILs via anion exchange for the tosylate counter ions of imidazolium cations in the inner ionic hyperPIL shell.

Going beyond the shuttling of anionic organic dyes, the switchable hyperPILs also turned out to be effective with respect to shuttling anionic palladium complexes (see Figure 6). The watersoluble palladium complex used was formed from the dipotassium salt of the triphenylphosphine ligand $\operatorname{bis}(p$-sulfonatophenyl)phenylphosphine and disodium tetrachloropalladate (see Figure S20 for proposed structure of the complex). Similar to the transport of Congo red, PEHO-IL$\mathrm{P} n \mathrm{PrOx}_{10}$ was used as thermoresponsive shuttle. At $0{ }^{\circ} \mathrm{C}$, the $\mathrm{Pd}$ complex was dissolved in the aqueous phase. Upon heating to $70^{\circ} \mathrm{C}$, the hyperPIL migrated into the ethyl acetate phase 
together with the palladium complex payload. By cooling to $0{ }^{\circ} \mathrm{C}$, the complex was transported back from the ethyl acetate phase to the aqueous phase. Just like the Congo red transport, this process was repeated several times without impairing shuttling. Hence, PEHO-IL-P $n \operatorname{PrOx}_{10}$ serves as shuttle for both organic and inorganic anions.
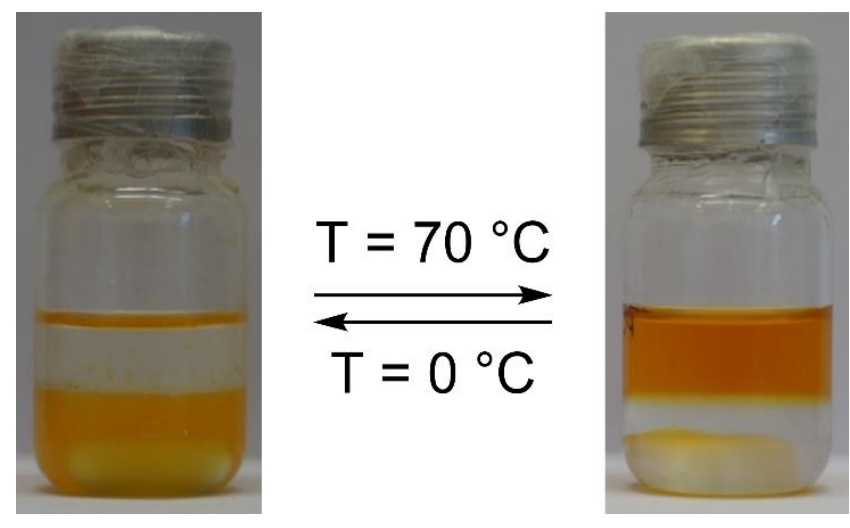

Figure 6: PEHO-IL-P $n \operatorname{PrOx}_{10}$ as thermoresponsive shuttle for an anionic water-soluble Pdphosphine complex in the ethyl acetate/water two-phase system.

\section{CONCLUSION}

Polyfunctional polyelectrolyte carriers and molecular shuttles for anionic organic and inorganic payloads are readily tailored via the chain termination of the cationic 2-oxazoline ringopening polymerization with imidazole-terminated hyperbranched poly(3-ethyl-3hydroxymethyloxetane) (PEHO-Im). In this efficient "grafting-to" process, the cationic oxazolinium end groups of the poly(2-alkyl-2-oxazoline)s alkylate the imidazole groups of PEHO-Im to produce cationic alkyl imidazolium coupling groups as IL moieties incorporated within the hyperbranched polyelectrolyte backbone. The resulting hyperbranched polymer ionic liquids (hyperPILs) exhibit nanostructures resembling micelles but are much more robust with respect to their thermal and chemical stabilities as well as their low shear and $\mathrm{pH}$ sensitivity. The covalent attachment of LCST-polyoxazolines derived from 2-ethyl- and 2-n-propyl-2-oxazoline renders hyperPILs thermoresponsive and enables the programmable thermal switching of the 
hyperPIL polarity and its hydrophilicity/hydrophobicity balance. The monomer type, the polyoxazoline chain length and copolymer compositions govern the cloud point $\left(T_{\mathrm{CP}}\right)$ of hyperPILs in water, which varies from 21 to $93{ }^{\circ} \mathrm{C}$. As verified by the addition of an anionic organic dye (Congo red), both hyperPILs and dye molecules are shuttled back and forth between water and ethyl acetate phases at temperatures below and above the corresponding $T_{\mathrm{CP}}$. In contrast, substitution of LCST-polyoxazolines by long-chain alkyl groups exclusively permits irreversible temperature-independent dye transport in one direction from water to the organic phase. Since shuttling in water/ethyl acetate is achieved only by using anionic dyes, it appears highly likely that anionic dyes are immobilized on hyperPILs via anion exchange for tosylate counter ions of the imidazolium moieties. Successful shuttling in water/ethyl acetate in the presence of hyperPILs is also demonstrated for inorganic anions such as palladium complexes containing $\operatorname{bis}(p$-sulfonatophenyl)phenylphosphine as a ligand. In principle, this thermal switching process using programmable thermoresponsive hyperPILs as polyfunctional carriers for anionic payloads holds great prospects with respect to the facile recovery of homogeneous catalysts. Upon completing the catalytic reaction in an organic medium at a temperature above $T_{\mathrm{CP}}$, the temperature is lowered to recover the catalyst by extracting it with water. It is then reused by the subsequent shuttling back into an organic phase at elevated temperature. 


\section{EXPERIMENTAL SECTION}

Materials. 3-Ethyl-3-hydroxymethyloxetane (EHO) was kindly provided by Perstorp Polyols. $\mathrm{BF}_{3} \cdot \mathrm{OEt}_{2}$ and 2-ethyl-2-oxazoline (99\%) were purchased from Alfa Aesar. Trifluoroacetic anhydride (99\%), tosyl chloride (98\%), methyl tosylate (98\%), and Congo red were obtained from Merck. Imidazole (99\%), $\mathrm{Na}_{2} \mathrm{PdCl}_{4}(98 \%)$, and bis( $p$-sulfonatophenyl)phenylphosphine dihydrate dipotassium salt (97\%) were purchased from Sigma-Aldrich. 2-n-Propyl-2-oxazoline was synthesized according to a literature procedure. ${ }^{42}$ Solvents were of analytical grade and purchased from different suppliers. DMF, 2-ethyl-2-oxazoline and 2-n-propyl-2-oxazoline were distilled and stored over molecular sieves ( $4 \AA$ ) prior to use. All other chemicals were used as received.

General remarks. All syntheses were performed in dry conditions and under a nitrogen atmosphere using standard Schlenk techniques. In the calculation of the end group equivalents of the PEHO derivatives, the degrees of functionalization determined from the respective ${ }^{1} \mathrm{H}$ NMR spectra were considered.

Synthesis of PEHO-OH. To a solution of $\mathrm{BF}_{3} \cdot \mathrm{OEt}_{2}(5.5 \mathrm{~mL}, 6.2 \mathrm{~g}, 44 \mathrm{mmol}, 1.0$ equiv. $)$ in dry $\mathrm{CH}_{2} \mathrm{Cl}_{2}(100 \mathrm{~mL})$ 3-ethyl-3-hydroxymethyloxetane (50 mL, $51 \mathrm{~g}, 0.44 \mathrm{~mol}, 10$ equiv.) was added dropwise under cooling in a water bath over a period of $2 \mathrm{~h}$. Then, the reaction mixture was stirred at room temperature for $48 \mathrm{~h}$. Afterwards, the reaction was stopped by addition of ethanol $(10 \mathrm{~mL})$ and the mixture was concentrated under reduced pressure until it became highly viscous but was still pourable. Subsequently, the mixture was precipitated in cyclohexane. The precipitate was dissolved in ethanol $(75 \mathrm{~mL})$ under gentle heating and the resulting polymer solution was precipitated in water $(300 \mathrm{~mL})$. The solid part was first dried at $60{ }^{\circ} \mathrm{C}$ in an oven 
and then at $80^{\circ} \mathrm{C}$ under high vacuum. The product was obtained as a colorless solid (48.4 g; $95 \%)$.

Synthesis of PEHO-TFA. A solution of PEHO-OH (474 mg, $4.08 \mathrm{mmol} \mathrm{OH}$ groups, 1.0 equiv.) in trifluoroacetic anhydride $(10 \mathrm{~mL}, 15 \mathrm{~g}, 71 \mathrm{mmol}, 17$ equiv.) was stirred under reflux for $4 \mathrm{~h}$. Excess trifluoroacetic anhydride and formed trifluoroacetic acid were distilled off and the remaining highly viscous liquid was dried at $70{ }^{\circ} \mathrm{C}$ under high vacuum. A colorless liquid (872 mg; $100 \%$ ) was obtained.

Synthesis of PEHO-OTs. To a solution of PEHO-OH $(25.0 \mathrm{~g}, 215 \mathrm{mmol} \mathrm{OH}$ groups, 1.0 equiv.) in pyridine $(125 \mathrm{~mL})$, a solution of $p$-tosyl chloride $(82.0 \mathrm{~g}, 430 \mathrm{mmol}, 2.0 \mathrm{equiv}$.$) in$ pyridine $(63 \mathrm{~mL})$ was added at $0{ }^{\circ} \mathrm{C}$ over a period of $30 \mathrm{~min}$. The reaction mixture was then stirred at room temperature for $7 \mathrm{~d}$. Toluene $(63 \mathrm{~mL})$ was added to the reaction solution and the resulting precipitates were filtered off. Then, saturated aqueous $\mathrm{K}_{2} \mathrm{CO}_{3}(63 \mathrm{~mL})$ was added portion wise to the filtrate. The aqueous phase was separated and the organic phase was concentrated under reduced pressure. The residue was taken up in $\mathrm{CHCl}_{3}(200 \mathrm{~mL})$ and washed with water $(2 \times 200 \mathrm{~mL})$, aqueous $\mathrm{HCl}(10 \%, 2 \times 200 \mathrm{~mL})$, water $(2 \times 200 \mathrm{~mL})$, and saturated aqueous $\mathrm{NaCl}(200 \mathrm{~mL})$. The organic phase was dried over $\mathrm{Na}_{2} \mathrm{SO}_{4}$ and concentrated under reduced pressure. The residue was dried at $80^{\circ} \mathrm{C}$ under high vacuum. PEHO-OTs was obtained as a slightly yellow solid (52.8 $\mathrm{g} ; 91 \%)$.

Synthesis of PEHO-Im. A mixture of PEHO-OTs (10.6 g, 37.4 mmol OTs groups, 1.0 equiv.) and imidazole $\left(55.3 \mathrm{~g}, 826 \mathrm{mmol}, 22\right.$ equiv.) was heated to $120{ }^{\circ} \mathrm{C}$, causing the imidazole to melt. The mixture was stirred at $120{ }^{\circ} \mathrm{C}$ for $24 \mathrm{~h}$. After cooling, the solidified mixture was dissolved in $\mathrm{CH}_{2} \mathrm{Cl}_{2}(200 \mathrm{~mL})$ and the resulting solution was washed with aqueous $\mathrm{NaOH}(2 \mathrm{M}$, $2 \times 200 \mathrm{~mL})$, water $(200 \mathrm{~mL})$, and saturated aqueous $\mathrm{NaCl}(200 \mathrm{~mL})$. The organic phase was 
dried over $\mathrm{MgSO}_{4}$ and concentrated under reduced pressure. The residue was dried at $100{ }^{\circ} \mathrm{C}$ under high vacuum to yield PEHO-Im as a slightly brown solid (6.38 g; $90 \%)$.

Synthesis of the hyperPILs. The syntheses of the hyperPILs were performed according to the following general procedure (weights, volumes and durations of polymerizations are listed in Table S1). To a solution of MeOTs (1.0 equiv.) in DMF the oxazoline monomer (10 or 20 equiv.) was added and the reaction solution was stirred in a preheated oil bath at $90{ }^{\circ} \mathrm{C}$ until the monomer was fully consumed. A solution of PEHO-Im (0.9 equiv. imidazole groups) in DMF was then added and stirred at $90{ }^{\circ} \mathrm{C}$ for another $20 \mathrm{~h}$. After cooling, the solution was precipitated in $\mathrm{Et}_{2} \mathrm{O}$ (3 times the volume of the reaction mixture). The precipitate was dissolved in $\mathrm{CH}_{2} \mathrm{Cl}_{2}$ (3 $\mathrm{mL}$ per gram of precipitate) and again precipitated in $\mathrm{Et}_{2} \mathrm{O}$ (10 times the volume of the $\mathrm{CH}_{2} \mathrm{Cl}_{2}$ solution). The precipitation was performed one more time. The obtained precipitate was filtered off and dried under high vacuum. In the case of PEHO-IL-PEtOx ${ }_{10}$, PEHO-IL$\mathrm{PEtOx}_{20}$, and PEHO-IL-P(EtOx/ $\left.n \mathrm{PrOx}\right)_{20}$, after precipitation in $\mathrm{Et}_{2} \mathrm{O}$ the hyperPILs, according to SEC analysis, still contained a considerable amount of low-molecular-weight free polyoxazolines. Therefore, a temperature-induced phase separation (TIPS) procedure was performed for further purification. For this purpose, the polymer to be purified was dissolved in water $\left(\mathrm{c}=100 \mathrm{~g} \mathrm{~L}^{-1}\right)$ and heated in a closed vessel slightly above $T_{\mathrm{CP}}$ for $24 \mathrm{~h}$ (see Table $\mathrm{S} 2$ and S3). As a result two phases formed. The upper phase contained the main part of the free polyoxazolines, the lower phase the hyperPIL in purified form. The upper phase was pipetted off and the solvent was removed from both phases by freeze-drying. Yields and purities of the hyperPILs, which were obtained as white or yellow solids, are listed in Table S4.

HyperPIL-mediated temperature-switchable phase transfer of Congo red. Solutions of Congo red in water $\left(c=0.3 \mathrm{~g} \mathrm{~L}^{-1}\right)$ and of PEHO-IL-P $n \operatorname{PrOx}_{10}$ in EtOAc $\left(c=0.35 \mathrm{~g} \mathrm{~L}^{-1}\right)$ were 
prepared. $2 \mathrm{~mL}$ of each of the solutions were mixed, resulting in a two-phase system consisting of a colorless upper EtOAc phase and a red lower aqueous phase. In a closed vessel, the twophase system was then kept at $60{ }^{\circ} \mathrm{C}$ for $23 \mathrm{~h}$ in an oven. As a result, the upper EtOAc phase was colored red, whereas the lower aqueous phase was almost completely colorless. The two-phase system was then kept in a refrigerator at $4{ }^{\circ} \mathrm{C}$ for $18 \mathrm{~h}$, whereupon the pattern was reversed. The EtOAc phase was almost colorless and the aqueous phase was colored red. This process was repeated several times with the same observation.

HyperPIL-mediated temperature-switchable phase transfer of a Pd complex. A solution of bis( $p$-sulfonatophenyl)phenylphosphine dihydrate dipotassium salt $(13 \mathrm{mg}, 0.036 \mathrm{mmol}$, 3.0 equiv.) and disodium tetrachloropalladate $(3.5 \mathrm{mg}, 0.012 \mathrm{mmol}, 1.0$ equiv.) in degassed water $(3 \mathrm{~mL})$ was prepared under nitrogen atmosphere. This solution was let to stand at room temperature for $8 \mathrm{~d}$ to ensure complete complex formation. In addition, a solution of PEHO-IL-P $n$ PrOx 10 in degassed water $\left(\mathrm{c}=1.5 \mathrm{~g} \mathrm{~L}^{-1}\right)$ was prepared. A part of the Pd complex solution $(0.25 \mathrm{~mL})$ and a part of the polymer solution $(2.25 \mathrm{~mL})$ were mixed and degassed EtOAc $(2.5 \mathrm{~mL})$ was added. At this stage, the EtOAc phase was clear and colorless, while the aqueous phase was orange. The mixture was heated at $70{ }^{\circ} \mathrm{C}$ in an oil bath for 100 min under vigorous stirring. After letting the mixture rest for a short time for phase separation, a completely decolorized aqueous phase and an orange colored EtOAc phase were obtained. Then, the mixture was cooled to $0{ }^{\circ} \mathrm{C}$ in an ice bath, stirred vigorously for $30 \mathrm{~min}$ and let to rest for $35 \mathrm{~min}$ without stirring for phase separation. By this means, the state before heating was restored. The aqueous phase was colored orange and the EtOAc phase was colorless. This heating-cooling cycle was repeated several times with the same observation. 


\section{ANALYSIS AND CHARACTERIZATION}

NMR. NMR spectra were generally recorded in deuterated DMSO at $110^{\circ} \mathrm{C}$ on a Bruker Avance III HD $500 \mathrm{MHz}$ spectrometer (for ${ }^{1} \mathrm{H}$ ) or at $27^{\circ} \mathrm{C}$ on a Bruker Avance II $300 \mathrm{MHz}$ spectrometer (for ${ }^{13} \mathrm{C}$ ). As an exception, PEHO-TFA was measured in deuterated chloroform at $27{ }^{\circ} \mathrm{C}$ on a Bruker Avance II $300 \mathrm{MHz}$ spectrometer $\left({ }^{1} \mathrm{H}\right.$ and ${ }^{13} \mathrm{C}$ IG). The chemical shifts were referenced by the solvent signal.

SEC. SEC measurement of PEHO-OTs was performed on an Agilent 1260 Infinity System with RI detector and chloroform as eluent at a flow rate of $1 \mathrm{~mL} \mathrm{~min}^{-1}$ and a temperature of $22{ }^{\circ} \mathrm{C}$. The injection volume was $100 \mu \mathrm{L}$. SDV columns $(5 \mu \mathrm{m})$ by PSS were used: 1 x precolumn $(8 * 50 \mathrm{~mm}), 1 \times 1000 \AA, 1 \times 10000 \AA, 1$ x $100000 \AA$ and 1 x $1000000 \AA$ (each $8 * 300 \mathrm{~mm}$ ). For calibration, narrow PS standards (from PSS) in the mass range $162-$ $1800000 \mathrm{~g} \mathrm{~mol}^{-1}$ were used. Toluene was used as internal standard.

SEC measurements of the hyperPILs were performed on an Agilent 1260 Infinity System with RI detector and $\mathrm{DMAc} / \mathrm{LiBr}(0.5 \mathrm{wt} . \%)$ as eluent at a flow rate of $1 \mathrm{~mL} \mathrm{~min}^{-1}$ and a temperature of $50{ }^{\circ} \mathrm{C}$. The injection volume was $50 \mu \mathrm{L}$. GRAM columns $(10 \mu \mathrm{m})$ by PSS were used: 1 x precolumn $(8 * 80 \mathrm{~mm}), 1$ x $30 \AA$ und 2 x $1000 \AA$ (each $8 * 300 \mathrm{~mm})$. For calibration, narrow PMMA standards (from PSS) in the mass range $410-988000 \mathrm{~g} \mathrm{~mol}^{-1}$ were used. To determine the average molecular weights and dispersities of the hyperPILs, the high-molecular weight peak was evaluated, in case of the presence of minor low-molecular-weight impurities (the lower integration limit was chosen from the minimum between the two peaks).

TGA. TGA curves were recorded on a STA 409 or a STA 449F5 from Netzsch with a heating rate of $10 \mathrm{~K} \mathrm{~min}^{-1}$. To characterize the mass loss curves, the onset temperatures $\left(T_{\mathrm{O}}\right)$ were determined. 
DSC. DSC curves were recorded on a DSC Seiko 6200 with heating and cooling rates of $10 \mathrm{~K} \mathrm{~min}^{-1}$. The glass transition temperatures $\left(T_{\mathrm{g}}\right)$ were determined from the second heating curve using the inflection point of the transition.

Determination of $\boldsymbol{T}_{\mathbf{C P}}$. The transmission measurements were performed on photometers with Peltier element from Analytik Jena (S $300 \mathrm{VIS}$; for $T_{\mathrm{CP}}<60^{\circ} \mathrm{C}$ ) or ThermoFisher Scientific (Evolution 201; for $T_{\mathrm{CP}}>60^{\circ} \mathrm{C}$ ) at a wavelength of $500 \mathrm{~nm}$ and a heating/cooling rate of $1 \mathrm{~K} \mathrm{~min}^{-1}$. The reference was demineralized water at $20^{\circ} \mathrm{C}$. The inflection point of the transmission-temperature curve was used to determine $T_{\mathrm{CP} .}{ }^{45}$

TEM. TEM images were captured with a FEI Talos L120C transmission electron microscope. The samples were prepared at room temperature by dropcasting of clear 1 wt.\% aqueous hyperPIL solutions on a 200 mesh carbon-coated copper grid (Science Services, Munich, Germany) and were stained with aqueous uranyl acetate solution.

\section{ACKNOWLEDGMENTS}

The authors gratefully acknowledge financial support from the German Research Foundation (DFG) in the framework of the IRTG Soft Matter Science (GRK 1642). The authors thank Tobias Michael Trötschler for helpful discussions, Ivonne Knauer, Kristin Anne Lehmann and Carolin Daniela Guth for assistance in laboratory work, Andreas Warmbold for TGA \& DSC measurements, Marina Hagios for SEC measurements, Dr. Victor Pacheco Torres as well as Dr. Manfred Keller for NMR measurements, and Prof. Philipp Kurz for the possibility to use spectroscopic equipment for turbidity measurements. 


\section{REFERENCES}

(1) Micelles: Structural biochemistry, formation and functions and usage; Bradburn, D.; Bittinger, T., Eds.; Biochemistry Research Trends; Nova Science Publishers, Inc: New York, 2014.

(2) Dreiss, C. A.; Feng, Y. Wormlike Micelles; Royal Society of Chemistry: Cambridge, 2017.

(3) Feng, Y.; Chu, Z.; Dreiss, C. A. Smart wormlike micelles: Design, characteristics and applications; SpringerBriefs in molecular science; Springer: Heidelberg, 2015.

(4) La Sorella, G.; Strukul, G.; Scarso, A. Recent advances in catalysis in micellar media. Green Chem. 2015, 17, 644-683, DOI: 10.1039/C4GC01368A.

(5) Moroi, Y. Micelles; Springer US: Boston, MA, 1992.

(6) Moorefield, C. N.; Newkome, G. R. Unimolecular micelles: supramolecular use of dendritic constructs to create versatile molecular containers. C. R. Chimie 2003, 6, 715-724, DOI: 10.1016/j.crci.2003.04.002.

(7) Kohno, Y.; Saita, S.; Men, Y.; Yuan, J.; Ohno, H. Thermoresponsive polyelectrolytes derived from ionic liquids. Polym. Chem. 2015, 6, 2163-2178, DOI: 10.1039/C4PY01665C.

(8) Kohno, Y.; Ohno, H. Key Factors to Prepare Polyelectrolytes Showing TemperatureSensitive Lower Critical Solution Temperature-type Phase Transitions in Water. Aust. J. Chem. 2012, 65, 91, DOI: 10.1071/CH11378. 
(9) Men, Y.; Li, X.-H.; Antonietti, M.; Yuan, J. Poly(tetrabutylphosphonium 4styrenesulfonate): A poly(ionic liquid) stabilizer for graphene being multi-responsive. Polym. Chem. 2012, 3, 871, DOI: 10.1039/c2py20011b.

(10) Kohno, Y.; Deguchi, Y.; Ohno, H. Ionic liquid-derived charged polymers to show highly thermoresponsive LCST-type transition with water at desired temperatures. Chemical communications (Cambridge, England) 2012, 48, 11883-11885, DOI: 10.1039/C2CC36913C.

(11) Kohno, Y.; Deguchi, Y.; Inoue, N.; Ohno, H. Temperature-Driven and Reversible Assembly of Homopolyelectrolytes Derived from Suitably Designed Ionic Liquids in Water. Aust. J. Chem. 2013, 66, 1393, DOI: 10.1071/CH13301.

(12) Men, Y.; Schlaad, H.; Yuan, J. Cationic Poly(ionic liquid) with Tunable Lower Critical Solution Temperature-Type Phase Transition. ACS Macro Lett. 2013, 2, 456-459, DOI: $10.1021 / \mathrm{mz} 400155 \mathrm{r}$.

(13) Men, Y.; Schlaad, H.; Voelkel, A.; Yuan, J. Thermoresponsive polymerized gemini dicationic ionic liquid. Polym. Chem. 2014, 5, 3719, DOI: 10.1039/c3py01790g.

(14) Mori, H.; Yahagi, M.; Endo, T. RAFT Polymerization of N -Vinylimidazolium Salts and Synthesis of Thermoresponsive Ionic Liquid Block Copolymers. Macromolecules 2009, 42, 8082-8092, DOI: 10.1021/ma901180j.

(15) Tauer, K.; Weber, N.; Texter, J. Core-shell particle interconversion with di-stimuliresponsive diblock copolymers. Chemical communications (Cambridge, England) 2009, 60656067, DOI: 10.1039/b912148j. 
(16) Soll, S.; Antonietti, M.; Yuan, J. Double Stimuli-Responsive Copolymer Stabilizers for Multiwalled Carbon Nanotubes. ACS Macro Lett. 2012, 1, 84-87, DOI: 10.1021/mz200042h.

(17) Mori, H.; Ebina, Y.; Kambara, R.; Nakabayashi, K. Temperature-responsive self-assembly of star block copolymers with poly(ionic liquid) segments. Polym $J$ 2012, 44, 550-560, DOI: 10.1038/pj.2012.35.

(18) Karjalainen, E.; Chenna, N.; Laurinmäki, P.; Butcher, S. J.; Tenhu, H. Diblock copolymers consisting of a polymerized ionic liquid and poly( $\mathrm{N}$-isopropylacrylamide). Effects of PNIPAM block length and counter ion on self-assembling and thermal properties. Polym. Chem. 2013, 4, 1014-1024, DOI: 10.1039/C2PY20815F.

(19) Men, Y.; Drechsler, M.; Yuan, J. Double-stimuli-responsive spherical polymer brushes with a poly(ionic liquid) core and a thermoresponsive shell. Macromolecular rapid communications 2013, 34, 1721-1727, DOI: 10.1002/marc.201300628.

(20) Wang, D.; Jin, Y.; Zhu, X.; Yan, D. Synthesis and applications of stimuli-responsive hyperbranched polymers. Progress in Polymer Science 2017, 64, 114-153, DOI: 10.1016/j.progpolymsci.2016.09.005.

(21) Korolovych, V. F.; Erwin, A.; Stryutsky, A.; Lee, H.; Heller, W. T.; Shevchenko, V. V.; Bulavin, L. A.; Tsukruk, V. V. Thermally Responsive Hyperbranched Poly(ionic liquid)s: Assembly and Phase Transformations. Macromolecules 2018, 51, 4923-4937, DOI: 10.1021/acs.macromol.8b00845.

(22) Schüler, F.; Kerscher, B.; Beckert, F.; Thomann, R.; Mülhaupt, R. Hyperbranched polymeric ionic liquids with onion-like topology as transporters and compartmentalized systems. 
Angew. Chem., 2013, 125, 473-476. Angew. Chem. Int. Ed. 2013, 52, 455-458, DOI: 10.1002/anie.201205130.

(23) Kerscher, B.; Schüler, F.; Evers, A.-K.; Mülhaupt, R. Chapter 7. Polymeric Ionic Liquids with Micelle-like Topologies and Functions. In Bio-inspired Polymers; Bruns, N., Kilbinger, A. F. M., Eds.; Polymer Chemistry Series; Royal Society of Chemistry: Cambridge, 2016; pp 259285.

(24) He, Y.; Lodge, T. P. The micellar shuttle: Thermoreversible, intact transfer of block copolymer micelles between an ionic liquid and water. J. Am. Chem. Soc. 2006, 128, 1266612667, DOI: $10.1021 / \mathrm{ja} 0655587$.

(25) Bai, Z.; He, Y.; Young, N. P.; Lodge, T. P. A Thermoreversible Micellization-Transfer-Demicellization Shuttle between Water and an Ionic Liquid. Macromolecules 2008, 41, 6615-6617, DOI: 10.1021/ma8011152.

(26) Bai, Z.; He, Y.; Lodge, T. P. Block copolymer micelle shuttles with tunable transfer temperatures between ionic liquids and aqueous solutions. Langmuir 2008, 24, 5284-5290, DOI: 10.1021/la703848e.

(27) Bai, Z.; Lodge, T. P. Thermodynamics and mechanism of the block copolymer micelle shuttle between water and an ionic liquid. J. Phys. Chem. B 2009, 113, 14151-14157, DOI: $10.1021 /$ jp907059q.

(28) Bai, Z.; Lodge, T. P. Pluronic micelle shuttle between water and an ionic liquid. Langmuir 2010, 26, 8887-8892, DOI: 10.1021/la9046462. 
(29) Guerrero-Sanchez, C.; Gohy, J.-F.; D'Haese, C.; Thijs, H.; Hoogenboom, R.; Schubert, U. S. Controlled thermoreversible transfer of poly(oxazoline) micelles between an ionic liquid and water. Chem. Commun. 2008, 2753-2755, DOI: 10.1039/b804179b.

(30) Guerrero-Sanchez, C.; Wouters, D.; Hoeppener, S.; Hoogenboom, R.; Schubert, U. S. Micellar dye shuttle between water and an ionic liquid. Soft Matter 2011, 7, 3827, DOI: 10.1039/c0sm01352h.

(31) Horton, J. M.; Bai, Z.; Jiang, X.; Li, D.; Lodge, T. P.; Zhao, B. Spontaneous phase transfer of thermosensitive hairy particles between water and an ionic liquid. Langmuir 2011, 27, 2019-2027, DOI: $10.1021 / 1 \mathrm{a} 1044706$.

(32) Horton, J. M.; Bao, C.; Bai, Z.; Lodge, T. P.; Zhao, B. Temperature- and pH-triggered reversible transfer of doubly responsive hairy particles between water and a hydrophobic ionic liquid. Langmuir 2011, 27, 13324-13334, DOI: 10.1021/la2031818.

(33) Bao, C.; Horton, J. M.; Bai, Z.; Li, D.; Lodge, T. P.; Zhao, B. Stimuli-Triggered Phase Transfer of Polymer-Inorganic Hybrid Hairy Particles Between Two Immiscible Liquid Phases. J. Polym. Sci., Part B: Polym. Phys. 2014, 52, 1600-1619.

(34) Li, D.; Zhao, B. Temperature-Induced Transport of Thermosensitive Hairy Hybrid Nanoparticles between Aqueous and Organic Phases. Langmuir 2007, 23, 2208-2217.

(35) Li, D.; Dunlap, J. R.; Zhao, B. Thermosensitive water-dispersible hairy particle-supported pd nanoparticles for catalysis of hydrogenation in an aqueous/organic biphasic system. Langmuir 2008, 24, 5911-5918, DOI: 10.1021/la800277j. 
(36) Kerscher, B. Nanostrukturierte polyionische Flüssigkeiten auf Basis hyperverzweigter Polyoxetane als Transporter, Dispergiermittel und Hybridmaterialien. Dissertation, AlbertLudwigs-Universität Freiburg, Freiburg, 2013.

(37) Kerscher, B.; Trötschler, T. M.; Pásztói, B.; Gröer, S.; Szabó, Á.; Iván, B.; Mülhaupt, R. Thermoresponsive Polymer Ionic Liquids and Nanostructured Hydrogels Based upon Amphiphilic Polyisobutylene-b-poly(2-ethyl-2-oxazoline) Diblock Copolymers.

Macromolecules 2019, 52, 3306-3318, DOI: 10.1021/acs.macromol.9b00296.

(38) Bednarek, M.; Biedron, T.; Helinski, J.; Kaluzynski, K.; Kubisa, P.; Penczek, S. Branched polyether with multiple primary hydroxyl groups: polymerization of 3-ethyl-3hydroxymethyloxetane. Macromol. Rapid Commun. 1999, 20, 369-372.

(39) Bednarek, M.; Kubisa, P.; Penczek, S. Multihydroxyl Branched Polyethers. 2. Mechanistic Aspects of Cationic Polymerization of 3-Ethyl-3-(hydroxymethyl)oxetane. Macromolecules 2001, 34, 5112-5119, DOI: 10.1021/ma002025o.

(40) Sudo, A.; Yamashita, H.; Endo, T. Ring-opening polymerization of 1,3-benzoxazines by p-toluenesulfonates as thermally latent initiators. J. Polym. Sci. A Polym. Chem. 2011, 49, 36313636, DOI: $10.1002 /$ pola.24801.

(41) Rettler, E. F.-J.; Kranenburg, J. M.; Lambermont-Thijs, H. M.L.; Hoogenboom, R.; Schubert, U. S. Thermal, Mechanical, and Surface Properties of Poly(2-N-alkyl-2-oxazoline)s. Macromol. Chem. Phys. 2010, 211, 2443-2448, DOI: 10.1002/macp.201000338. 
(42) Hoogenboom, R.; Fijten, M. W. M.; Thijs, H. M. L.; Lankvelt, B. M. van; Schubert, U. S. Microwave-assisted synthesis and properties of a series of poly(2-alkyl-2-oxazoline)s. Des. Monomers Polym. 2005, 8, 659-671, DOI: 10.1163/156855505774597704.

(43) Rosa, V. R. de la; Woisel, P.; Hoogenboom, R. Supramolecular control over thermoresponsive polymers. Mater. Today 2016, 19, 44-55, DOI: 10.1016/j.mattod.2015.06.013.

(44) Hoogenboom, R.; Thijs, H. M. L.; Jochems, M. J. H. C.; van Lankvelt, B. M.; Fijten, M. W. M.; Schubert, U. S. Tuning the LCST of poly(2-oxazoline)s by varying composition and molecular weight: Alternatives to poly(N-isopropylacrylamide)? Chem. Commun. 2008, 57585760, DOI: $10.1039 / \mathrm{b} 813140 \mathrm{f}$.

(45) Osváth, Z.; Iván, B. The Dependence of the Cloud Point, Clearing Point, and Hysteresis of Poly(N -isopropylacrylamide) on Experimental Conditions: The Need for Standardization of Thermoresponsive Transition Determinations. Macromol. Chem. Phys. 2017, 218, 1600470, DOI: 10.1002/macp.201600470. 


\section{Supporting Information}

\section{Programmable Thermoresponsive Micelle-Inspired Polymer Ionic Liquids as Molecular Shuttles for Anionic Payloads}

Steffen Wiedmann ${ }^{\dagger, \ddagger, \S}$, Manuel Luitz ${ }^{\dagger, \ddagger}, \perp$, Benjamin Kerscher ${ }^{\dagger, \neq}$, Jean-François Lutz"l, and Rolf Mülhaupt ${ }^{*,+, \neq, \S}$

${ }^{+}$Institute for Macromolecular Chemistry, University of Freiburg, Stefan-Meier-Str. 31, D-79104 Freiburg, Germany

${ }^{\ddagger}$ Freiburg Materials Research Center (FMF), University of Freiburg, Stefan-Meier-Str. 21, D-79104 Freiburg, Germany

${ }^{\S}$ Freiburg Centre for Interactive Materials and Bioinspired Technologies (FIT), University of Freiburg, Georges-Köhler-Allee 105, D-79110 Freiburg, Germany

"Institut Charles Sadron, CNRS, Université de Strasbourg, UPR22, 23 rue du Loess, 67034 Strasbourg Cedex 2, France

$\perp$ Present Address: Department of Microsystems Engineering (IMTEK), Laboratory of Process Technology, University of Freiburg, Georges-Köhler-Allee 103, D-79110 Freiburg, Germany 


\section{Table of Contents}

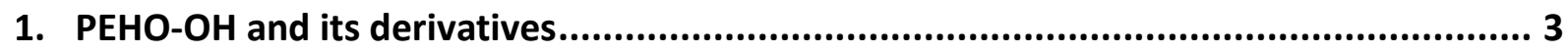

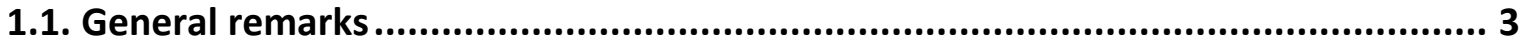

1.2. Structural characterization by NMR and SEC ................................................... 4

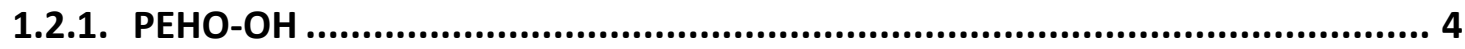

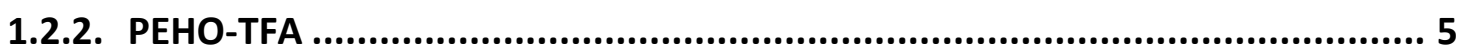

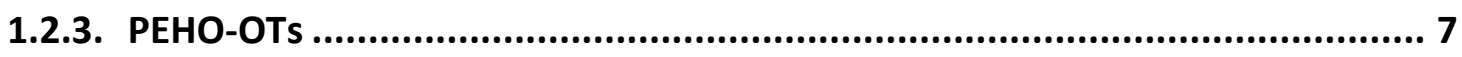

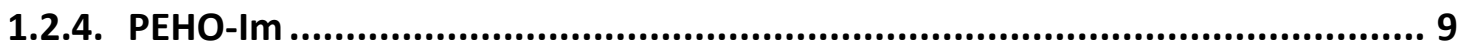

1.3. Thermal characterization by TGA and DSC ............................................................. 11

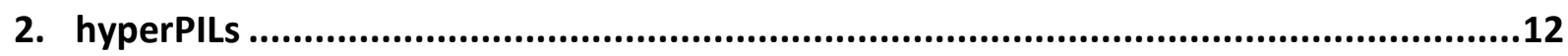

2.1. Listing of amounts and reaction times employed in syntheses ..........................12

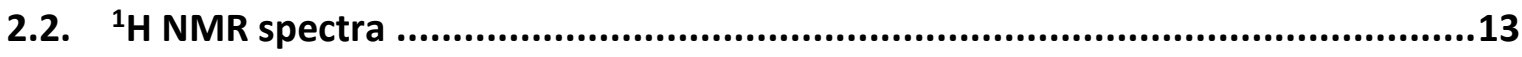

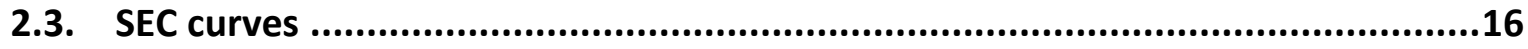

2.4. Listings of low-molecular-weight fractions, yields and purities .........................17

2.5. Procedure for calculating $M_{n}$ from the ${ }^{1} \mathrm{H}$ NMR spectra ................................19

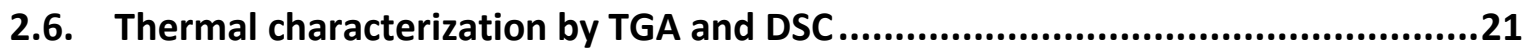

2.7. Transmissions-temperature curves............................................................22

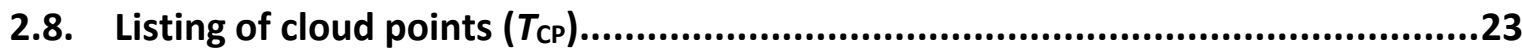

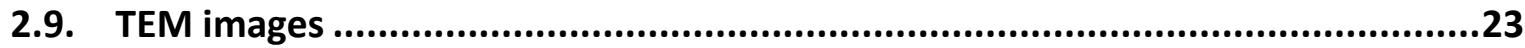

2.10. Chemical structures of the molecules used for the transport experiments .........24

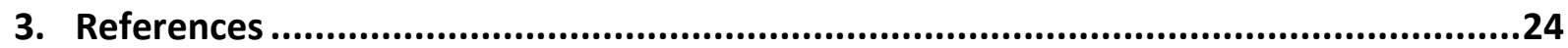




\section{PEHO-OH and its derivatives}

\subsection{General remarks}

For the sake of convenience, the structural formulas in the following NMR spectra only show the linear repeat unit of $\mathrm{PEHO}-\mathrm{OH}$ and its derivatives despite the hyperbranched architecture. To calculate the average molecular mass of a repeat unit of $\mathrm{PEHO}-\mathrm{OH}$ and its derivatives, the molecular masses of the linear repeat units were used in good approximation. In case of different end groups being present, weighting was based on the corresponding degree of functionalization (DF). This is demonstrated exemplary in the following equation for a PEHO derivative PEHO-B, which was obtained from PEHO-A by polymer analogous reaction and contains not just B groups, but also still a certain amount of non-reacted A groups.

$$
\overline{M_{\text {rep }}}(\text { PEHO-B })=[1-D F(P E H O-B)] * M(P E H O-A)+D F(\text { PEHO-B }) * M(P E H O-B)
$$

To calculate the equivalents of the functional end group $B$, the molar mass $M(B)$ was used, which indicates the mass of PEHO-B that contains one mole of B groups:

$$
M(B)=\frac{\overline{M_{r e p}}(P E H O-B)}{D F(P E H O-B)}
$$




\subsection{Structural characterization by NMR and SEC}

\subsubsection{PEHO-OH}
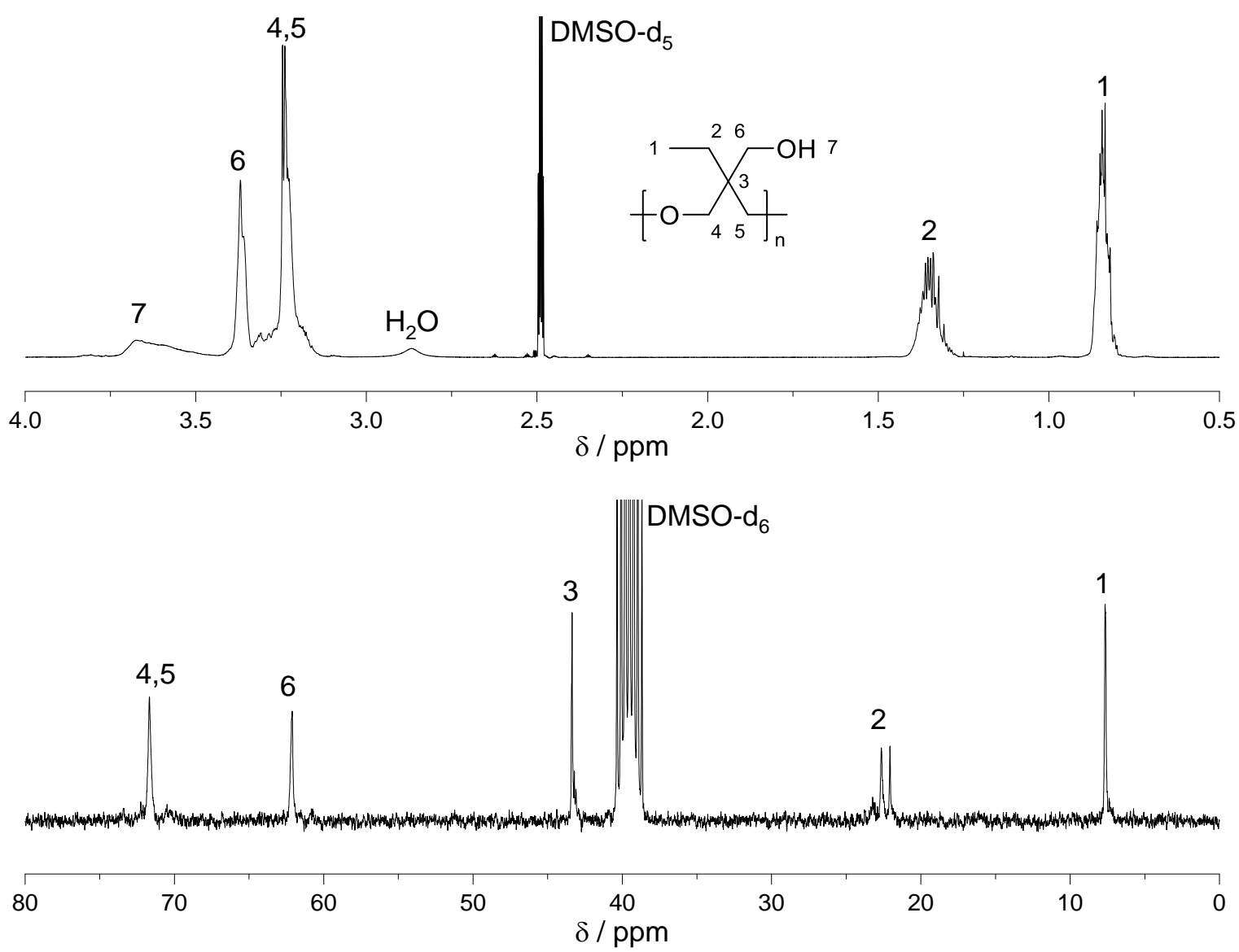

Figure S1: ${ }^{1} \mathrm{H}$ (top) and ${ }^{13} \mathrm{C}$ NMR spectrum (bottom) of $\mathrm{PEHO}-\mathrm{OH}$.

${ }^{1} \mathrm{H} N M R \quad(500 \mathrm{MHz}, \quad$ DMSO-d $6,383 \mathrm{~K}): \delta=0.75-0.92\left(3 \mathrm{H}, \mathrm{H}^{1}\right), \quad 1.26-1.43\left(2 \mathrm{H}, \mathrm{H}^{2}\right)$, $3.13-3.33\left(4 \mathrm{H}, \mathrm{H}^{4}+\mathrm{H}^{5}\right), 3.33-3.42\left(2 \mathrm{H}, \mathrm{H}^{6}\right), 3.44-3.75\left(1 \mathrm{H}, \mathrm{H}^{7}\right) \mathrm{ppm}$.

${ }^{13} \mathrm{C}$ NMR (75 MHz, DMSO-d $\left.\mathrm{d}_{6}, 300 \mathrm{~K}\right): \delta=7.66\left(\mathrm{C}^{1}\right), 22.07,22.65\left(\mathrm{C}^{2}\right), 43.46\left(\mathrm{C}^{3}\right), 62.11\left(\mathrm{C}^{6}\right), 71.68$ $\left(C^{4}+C^{5}\right) p p m$.

Since SEC measurements of PEHO-OH were not possible due to its limited solubility in the solvents commonly used for this purpose, $M_{n}$ was determined by multiplying the degree of polymerization (DP) determined for PEHO-OTs (see Section 1.2.3) with the molecular weight of a linear repeat unit of PEHO-OH.

$$
M_{n}(P E H O-O H)=D P * M_{r e p}(P E H O-O H)=15 * 116 \frac{\mathrm{g}}{\mathrm{mol}}=1700 \frac{\mathrm{g}}{\mathrm{mol}}
$$




\subsubsection{PEHO-TFA}
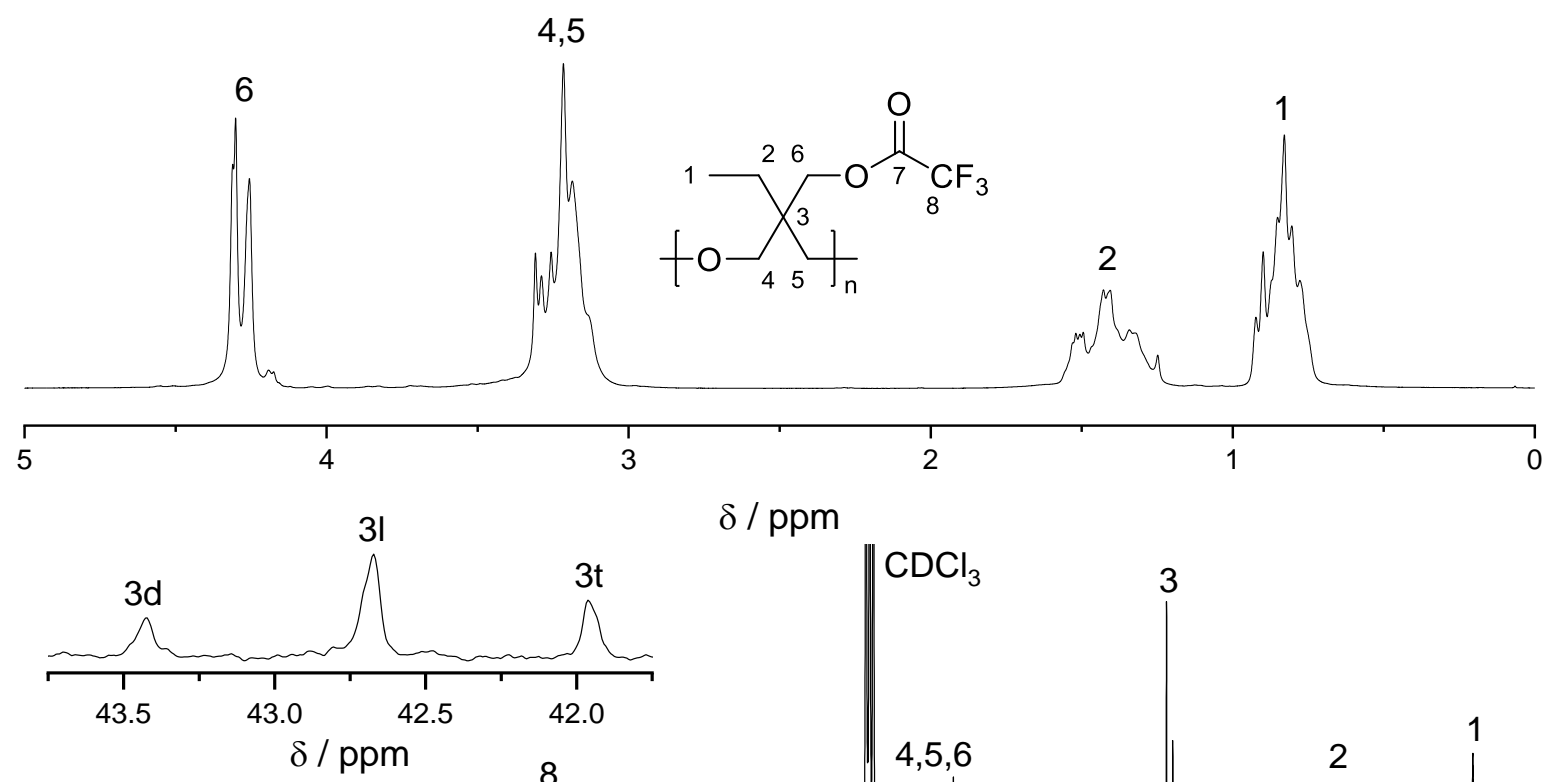

$\delta / \mathrm{ppm}$

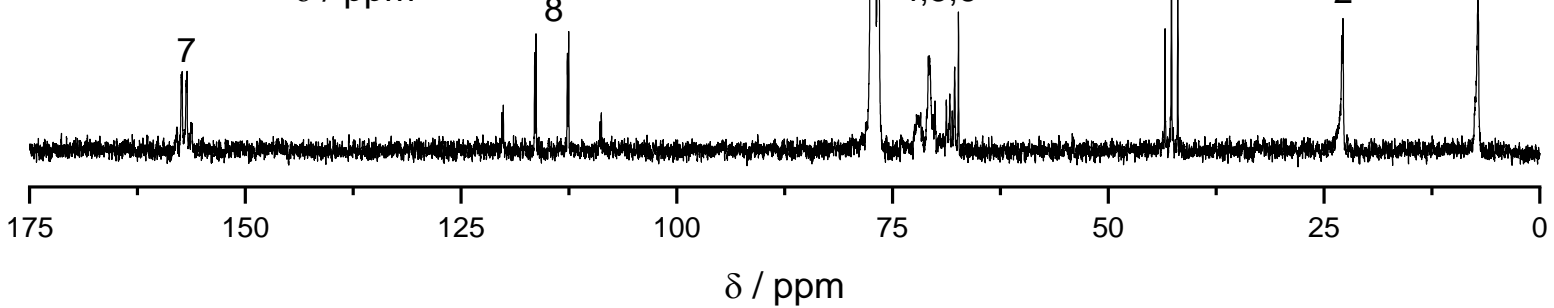

Figure S2: ${ }^{1} \mathrm{H}$ (top) and ${ }^{13} \mathrm{C}$ IG NMR spectrum (bottom, with magnification of signal 3) of PEHO-TFA. Assignment of the different structural units to the respective signals of $C^{3}$ is according to Bednarek et al. ${ }^{1}$

${ }^{1} \mathrm{H}$ NMR $\left(300 \mathrm{MHz}, \mathrm{CDCl}_{3}, 300 \mathrm{~K}\right): \delta=0.65-1.00\left(3 \mathrm{H}, \mathrm{H}^{1}\right), 1.18-1.61\left(2 \mathrm{H}, \mathrm{H}^{2}\right), 3.00-3.49(4 \mathrm{H}$, $\left.\mathrm{H}^{4}+\mathrm{H}^{5}\right), 4.11-4.47\left(2 \mathrm{H}, \mathrm{H}^{6}\right) \mathrm{ppm}$.

${ }^{13} \mathrm{C} \mathrm{IG-NMR}\left(75 \mathrm{MHz}, \mathrm{CDCl}_{3}, 300 \mathrm{~K}\right): \delta=7.11\left(\mathrm{C}^{1}\right), 22.72\left(\mathrm{C}^{2}\right), 41.89\left(\mathrm{C}^{3 \mathrm{t}}\right), 42.60\left(\mathrm{C}^{31}\right), 43.35\left(\mathrm{C}^{3 \mathrm{~d}}\right)$, $67.17-72.56\left(C^{4}+C^{5}+C^{6}\right), 114.42\left(q d,{ }^{1} J_{C, F}=286 \mathrm{~Hz}, 11 \mathrm{~Hz}, C^{8}\right), 156.99\left(q,{ }^{2} J_{C, F}=43 \mathrm{~Hz}, C^{7}\right)$ ppm.

Complete disappearance of the signal $\mathrm{H}^{6}$ of $\mathrm{PEHO}-\mathrm{OH}$ in the ${ }^{1} \mathrm{H}$ NMR spectrum of PEHO-TFA proves quantitative functionalization.

The proportion of the respective structural units $(d=$ dendritic, $I=$ linear, $t=$ terminal) was calculated from the integrals of the associated signals of $3 d(43.0-43.7 \mathrm{ppm}), 3 \mathrm{I}$ $(42.2-43.0 \mathrm{ppm}), 3 \mathrm{t}(41.5-42.2 \mathrm{ppm})$, and the total integral of all signals associated to $\mathrm{C}^{3}$ $(41.5-43.7 \mathrm{ppm})$ in the ${ }^{13} \mathrm{C}$ inverse-gated (IG) NMR spectrum. Signal assignment is according to Bednarek et al. ${ }^{1}$ 
This results in the following proportions of the structural units:

$d=23 \% ; l=53 \% ; t=24 \%$.

The degree of branching (DB) was calculated from the proportions of the structural units according to the equation of Frey et al. ${ }^{2}$ :

$$
D B=\frac{2 d}{2 d+l}=0.46
$$




\subsubsection{PEHO-OTS}
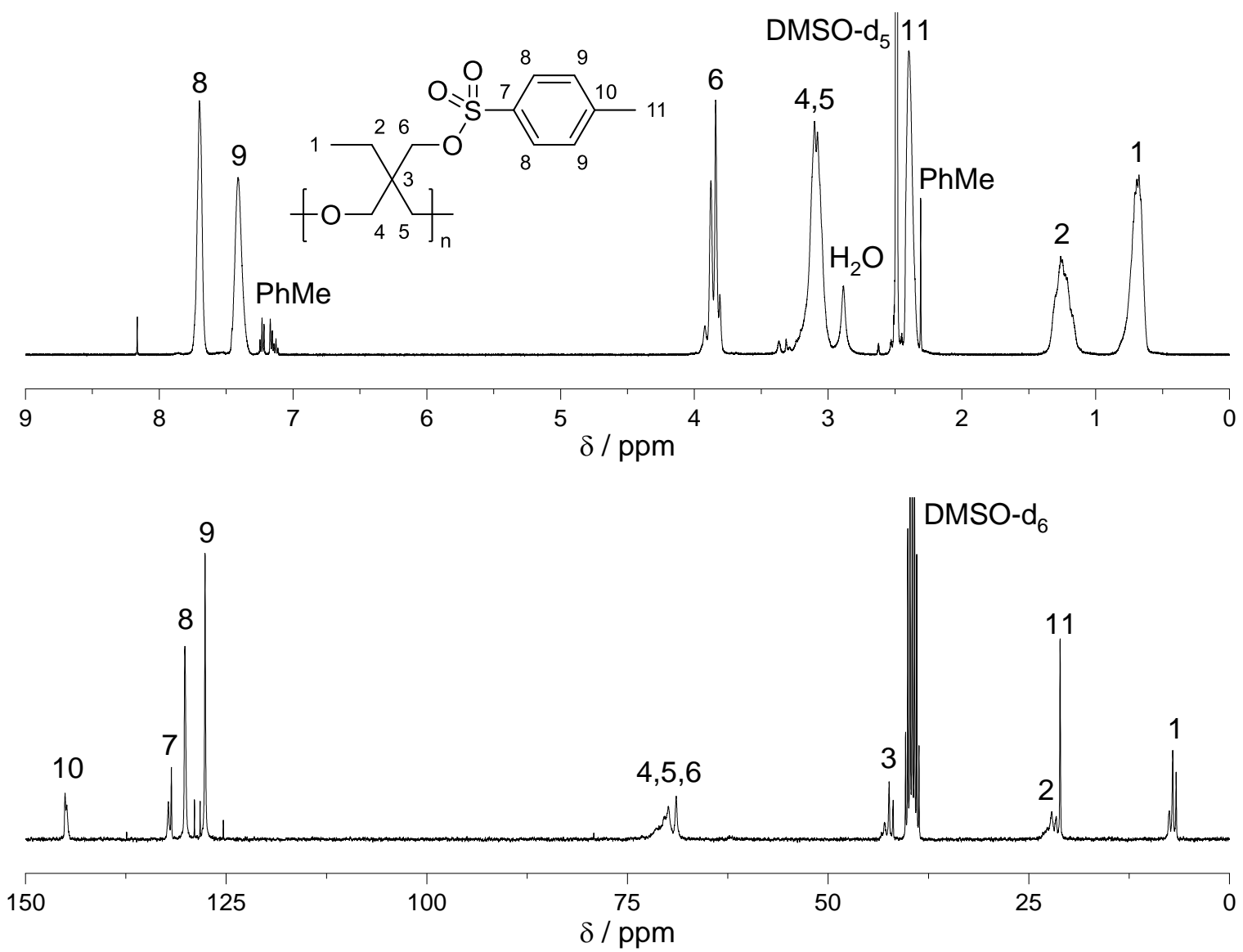

Figure S3: ${ }^{1} \mathrm{H}$ (top) and ${ }^{13} \mathrm{C}$ NMR spectrum (bottom) of PEHO-OTs.

${ }^{1} \mathrm{H} N M R \quad(500 \mathrm{MHz}$, DMSO-d $6,383 \mathrm{~K}): \delta=0.60-0.89\left(3 \mathrm{H}, \mathrm{H}^{1}\right), \quad 1.10-1.39\left(2 \mathrm{H}, \mathrm{H}^{2}\right)$, $2.30-2.44\left(3 \mathrm{H}, \mathrm{H}^{11}\right), 2.96-3.27\left(4 \mathrm{H}, \mathrm{H}^{4}+\mathrm{H}^{5}\right), 3.76-3.97\left(2 \mathrm{H}, \mathrm{H}^{6}\right), 7.30-7.48\left(2 \mathrm{H}, \mathrm{H}^{9}\right)$, $7.64-7.76\left(2 \mathrm{H}, \mathrm{H}^{8}\right) \mathrm{ppm}$.

${ }^{13} \mathrm{C}$ NMR (75 MHz, DMSO-d 6 , $\left.300 \mathrm{~K}\right): \delta=6.64,7.06,7.49\left(\mathrm{C}^{1}\right), 21.10\left(\mathrm{C}^{11}\right), 21.57,22.14\left(\mathrm{C}^{2}\right)$, 68.92, $69.91\left(C^{4}+C^{5}+C^{6}\right), 127.63\left(C^{9}\right), 130.14\left(C^{8}\right), 131.82,132.20\left(C^{7}\right), 145.07\left(C^{10}\right) p p m$.

The DF was determined from the ${ }^{1} \mathrm{H}$ NMR spectrum by comparing the integrals of the methyl groups of the PEHO core $\left(\mathrm{H}^{1}\right)$ with one of the two aromatic signals of the tosyl groups $\left(\mathrm{H}^{8}\right)$ according to the following equation.

$$
\text { DF (PEHO-OTS })=\frac{\left(\frac{I\left(H^{8}\right)}{2}\right)}{\left(\frac{I\left(H^{1}\right)}{3}\right)}=0.90
$$


Based on DF and, thus, the proportions of tosyl and hydroxy end groups, the average molar mass of a linear repeat unit of PEHO-OTs was calculated from the molar masses of the respective linear repeat units by weighting according to the following equation.

$M_{\text {rep }}(\mathrm{PEHO}-\mathrm{OH})=116 \mathrm{~g} \mathrm{~mol}^{-1}, M_{\text {rep }}(\mathrm{PEHO}-\mathrm{OTs})=270 \mathrm{~g} \mathrm{~mol}^{-1}, \mathrm{DF}=0.90$

$$
\begin{aligned}
\overline{M_{\text {rep }}}(\text { PEHO-OTs }) \\
=(1-\text { DF }(\text { PEHO-OTs })) * M_{\text {rep }}(\text { PEHO-OH }) \\
+ \text { DF }(\text { PEHO-OTs }) * M_{\text {rep }}(\text { PEHO-OTs }) \\
=0.10 * 116 \frac{\mathrm{g}}{\mathrm{mol}}+0.90 * 270 \frac{\mathrm{g}}{\mathrm{mol}}=255 \frac{\mathrm{g}}{\mathrm{mol}}
\end{aligned}
$$

The DP can be calculated from $M_{\mathrm{n}}$ determined by SEC through dividing it by the average molecular weight of a linear repeat unit.

$\operatorname{SEC}\left(\mathrm{CHCl}_{3}\right): M_{\mathrm{n}}=3800 \mathrm{~g} \mathrm{~mol}^{-1}, M_{\mathrm{w}}=7600 \mathrm{~g} \mathrm{~mol}^{-1}, Ð=2.0$

$$
D P=\frac{M_{n}(S E C)}{\overline{M_{\text {rep }}}}=\frac{3800 \frac{\mathrm{g}}{\mathrm{mol}}}{255 \frac{\mathrm{g}}{\mathrm{mol}}}=15
$$

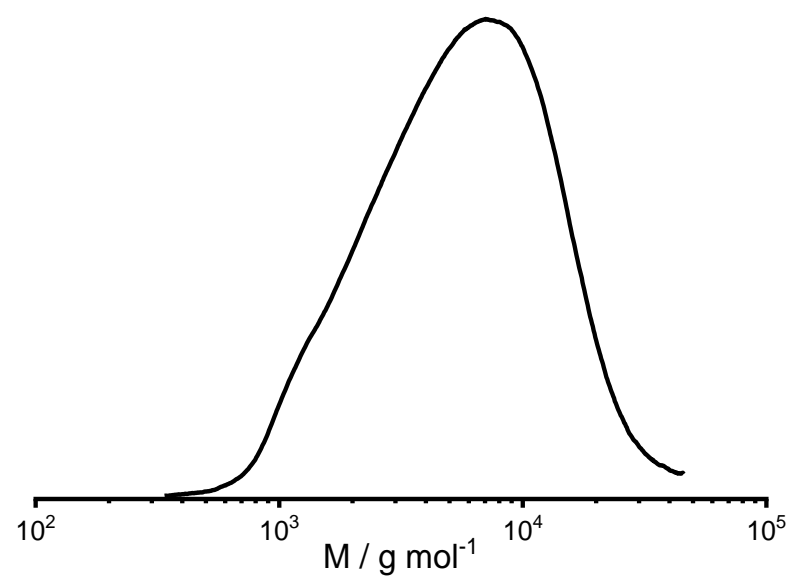

Figure S4: SEC curve of PEHO-OTs. 


\subsubsection{PEHO-Im}
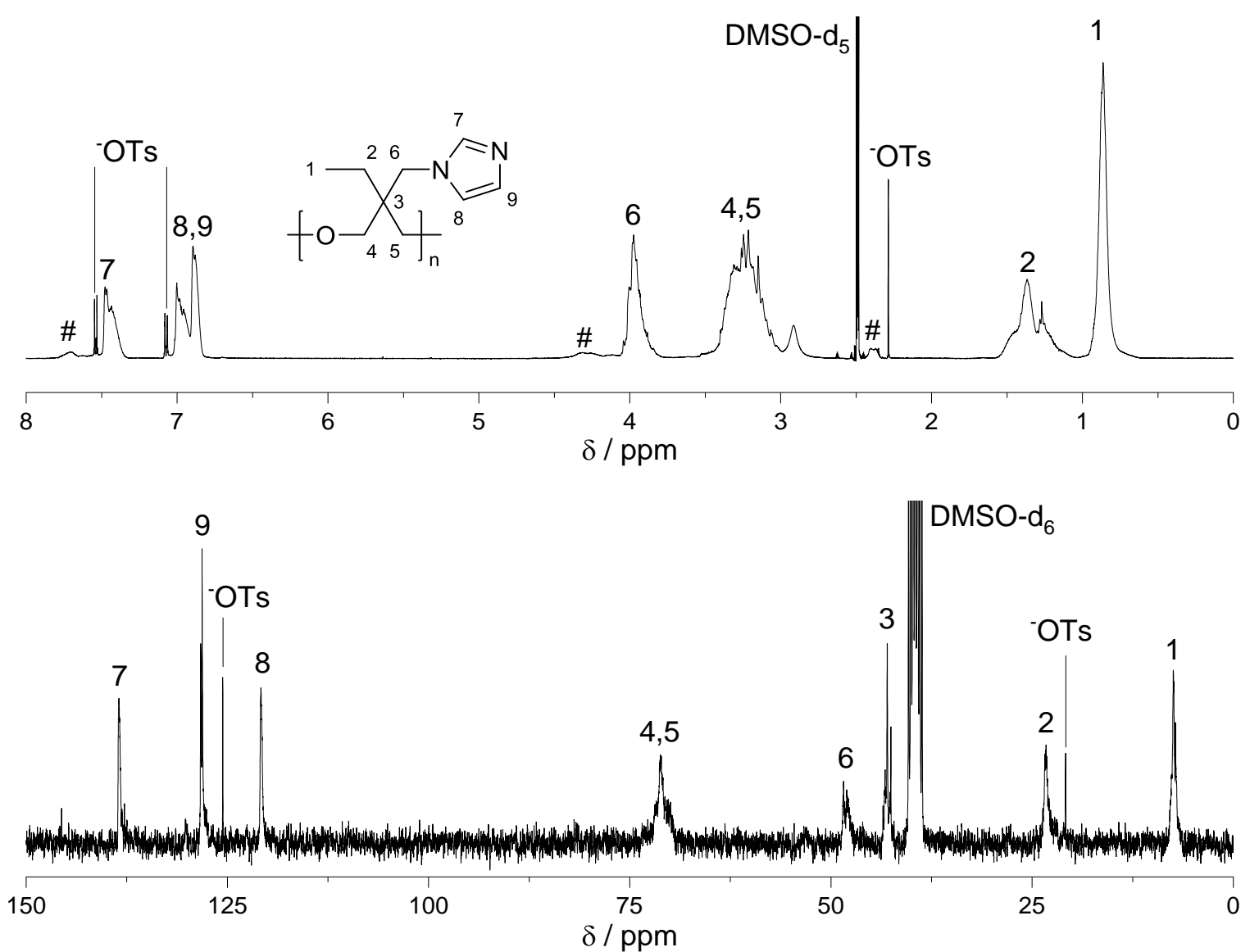

Figure S5: ${ }^{1 \mathrm{H}}$ (top) and ${ }^{13} \mathrm{C}$ NMR spectrum (bottom) of PEHO-Im. The signals marked with \# originate from unreacted OTs groups that are still bonded to the PEHO. The signals marked with -OTs, whose integral values are negligibly small, originate from free tosylate ions present as counterions to PEHO-bonded imidazolium units, which are formed to a small extent by presumably intramolecular dialkylation of imidazole groups.

${ }^{1} \mathrm{H} N M R \quad(500 \mathrm{MHz}$, DMSO-d $6,383 \mathrm{~K}): \delta=0.62-1.03\left(3 \mathrm{H}, \mathrm{H}^{1}\right), \quad 1.04-1.59\left(2 \mathrm{H}, \mathrm{H}^{2}\right)$, $2.99-3.54\left(3 \mathrm{H}, \mathrm{H}^{4}+\mathrm{H}^{5}\right), 3.76-4.06\left(4 \mathrm{H}, \mathrm{H}^{6}\right), 6.76-7.04\left(2 \mathrm{H}, \mathrm{H}^{8}+\mathrm{H}^{9}\right), 7.33-7.50\left(1 \mathrm{H}, \mathrm{H}^{7}\right)$ ppm.

${ }^{13} \mathrm{C}$ NMR (75 MHz, DMSO-d $\left.6,300 \mathrm{~K}\right): \delta=7.46\left(\mathrm{C}^{1}\right), 23.27\left(\mathrm{C}^{2}\right), 43.01\left(\mathrm{C}^{3}\right), 48.44\left(\mathrm{C}^{6}\right), 71.18\left(\mathrm{C}^{4}\right.$ $\left.+C^{5}\right), 120.83\left(C^{8}\right), 128.14\left(C^{9}\right), 138.48\left(C^{7}\right)$ ppm.

The DF was determined from the ${ }^{1} \mathrm{H}$ NMR spectrum by comparing the integrals of the methyl groups of the PEHO core $\left(\mathrm{H}^{1}\right)$ with one of the three aromatic signals of the imidazole groups $\left(\mathrm{H}^{7}\right)$ according to the following equation.

$$
D F(P E H O-I m)=\frac{\left(I\left(H^{7}\right)\right)}{\left(\frac{I\left(H^{1}\right)}{3}\right)}=0.82
$$


Based on proportions of hydroxy, tosyl, and imidazole end groups, the average molar mass of a linear repeat unit of PEHO-Im was calculated from the molar masses of the respective linear repeat units by weighting according to the following equation.

$M_{\text {rep }}\left(\right.$ PEHO-Im) $=166 \mathrm{~g} \mathrm{~mol}^{-1}$

$$
\begin{aligned}
\overline{M_{\text {rep }}}(\text { PEHO-Im }) \\
=(1-\text { DF }(\text { PEHO-OTs })) * M_{\text {rep }}(\text { PEHO-OH }) \\
+(\text { DF }(\text { PEHO-Im })-D F(\text { PEHO-OTs })) * M_{\text {rep }}(\text { PEHO-OTs }) \\
+ \text { DF }(\text { PEHO-Im }) * M_{\text {rep }}(\text { PEHO-Im }) \\
=0.10 * 116 \frac{\mathrm{g}}{\mathrm{mol}}+0.08 * 270 \frac{\mathrm{g}}{\mathrm{mol}}+0.82 * 166 \frac{\mathrm{g}}{\mathrm{mol}}=170 \frac{\mathrm{g}}{\mathrm{mol}}
\end{aligned}
$$

Since SEC measurements of PEHO-Im were not possible, $M_{\mathrm{n}}$ was determined by multiplying DP (obtained from the analysis of PEHO-OTs) with the average molecular weight of a repeat unit.

$$
M_{n}(P E H O-I m)=D P * \overline{M_{r e p}}(P E H O-I m)=15 * 170 \frac{g}{m o l}=2500 \frac{g}{m o l}
$$




\subsection{Thermal characterization by TGA and DSC}

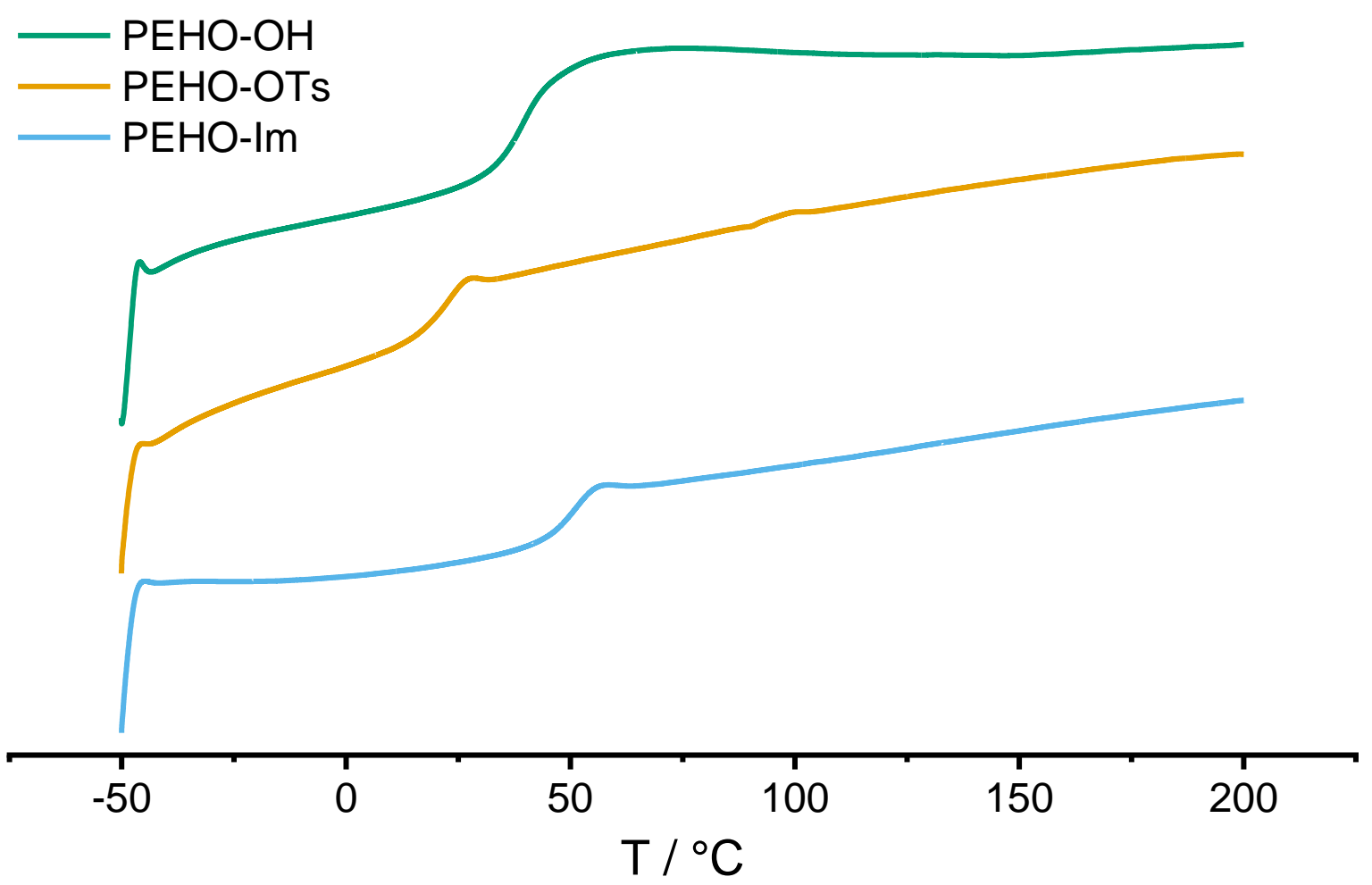

Figure S6: DSC thermograms ( $2^{\text {nd }}$ heating curve) of PEHO-OH and its derivatives.
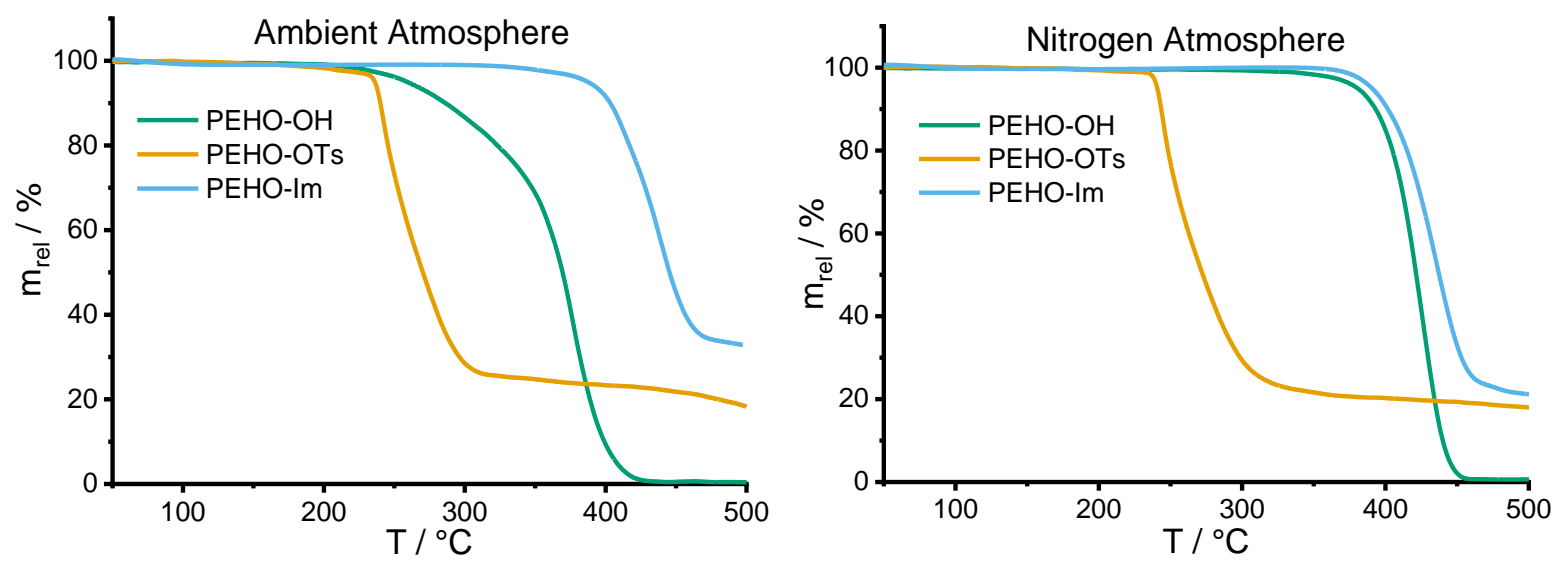

Figure S7: TGA curves of PEHO-OH and its derivatives measured under air (left) and nitrogen atmosphere (right). 


\section{2. hyperPILs}

\subsection{Listing of amounts and reaction times employed in syntheses}

Table S1: Weights, solvent volumes, and reaction times in hyperPIL syntheses.

\begin{tabular}{|c|c|c|c|c|c|c|c|}
\hline Sample code & $\begin{array}{c}\text { MeOTs } \\
\text { [mg | mmol | equiv.] }\end{array}$ & $\begin{array}{c}\text { 2-Ethyl-2-oxazoline } \\
{[\mathrm{mL}|\mathrm{g}| \mathrm{mmol} \mid \text { equiv. }]}\end{array}$ & $\begin{array}{l}\text { 2-n-Propyl-2-oxazoline } \\
{[\mathrm{mL}|\mathrm{g}| \mathrm{mmol} \mid \text { equiv.] }}\end{array}$ & $\begin{array}{l}\mathrm{DMF}^{\mathrm{a}} \\
{[\mathrm{mL}]}\end{array}$ & $\begin{array}{c}t \\
{[h]}\end{array}$ & $\begin{array}{c}\text { PEHO-Im } \\
{[\mathrm{mg}|\mathrm{mmol}| \text { equiv.] }}\end{array}$ & $\begin{array}{l}\mathrm{DMF}^{\mathrm{d}} \\
{[\mathrm{mL}]}\end{array}$ \\
\hline PEHO-IL-PEtOx 10 & $373|2.00| 1.0$ & $2.02|1.99| 20.0 \mid 10$ & - & 5.0 & 2 & $383|1.76| 0.9$ & 4.0 \\
\hline PEHO-IL-PEtOx 20 & $148|0.80| 1.0$ & $1.61|1.58| 15.9$ | 20 & - & 4.0 & 4 & $152|0.73| 0.9$ & 1.5 \\
\hline PEHO-IL-P(EtOx/nPrOx $)_{10}$ & $220|1.18| 1.0$ & $0.60|0.59| 5.91 \mid 5$ & $0.66|0.67| 5.91 \mid 5$ & 3.0 & 2 & 226 | 1.09 | 0.9 & 3.0 \\
\hline PEHO-IL-P(EtOx/nPrOx $)_{20}$ & $164|0.88| 1.0$ & $0.89|0.87| 8.80 \mid 10$ & $1.00|1.02| 8.80 \mid 10$ & 2.5 & 4 & $168|0.81| 0.9$ & 1.5 \\
\hline PEHO-IL-PnPrOx 10 & $360 \mid 1.94$ | 1.0 & - & 2.15 | 2.19 | 19.4 | & 5.0 & 2 & $374 \mid 1.81$ | 0.9 & 4.0 \\
\hline PEHO-IL-PnPrOx 20 & $238|1.28| 1.0$ & - & $2.83|2.89| 25.5 \mid 20$ & 6.5 & 4 & $245|1.18| 0.9$ & 3.0 \\
\hline
\end{tabular}

${ }^{a}$ Solvent volume for oxazoline polymerization; ${ }^{b}$ duration of oxazoline polymerization before addition of PEHO-Im; ${ }^{c}$ equivalents refer to imidazole groups; dsolvent volume of PEHO-Im solution. 


\section{2. ${ }^{1} \mathrm{H}$ NMR spectra}
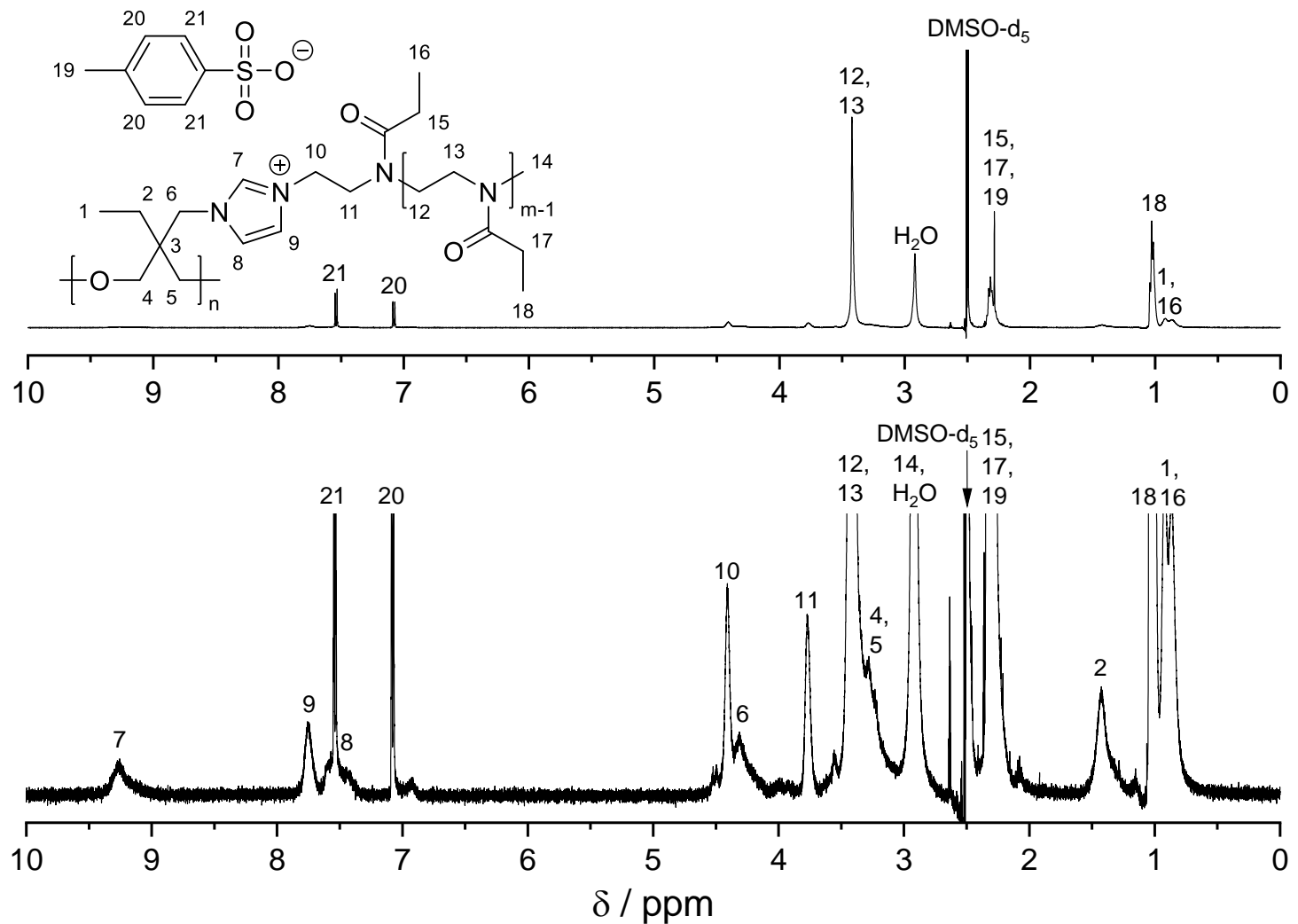

Figure S8: ${ }^{1} \mathrm{H}$ NMR spectrum of PEHO-IL-PEtOx 10 with magnification below.
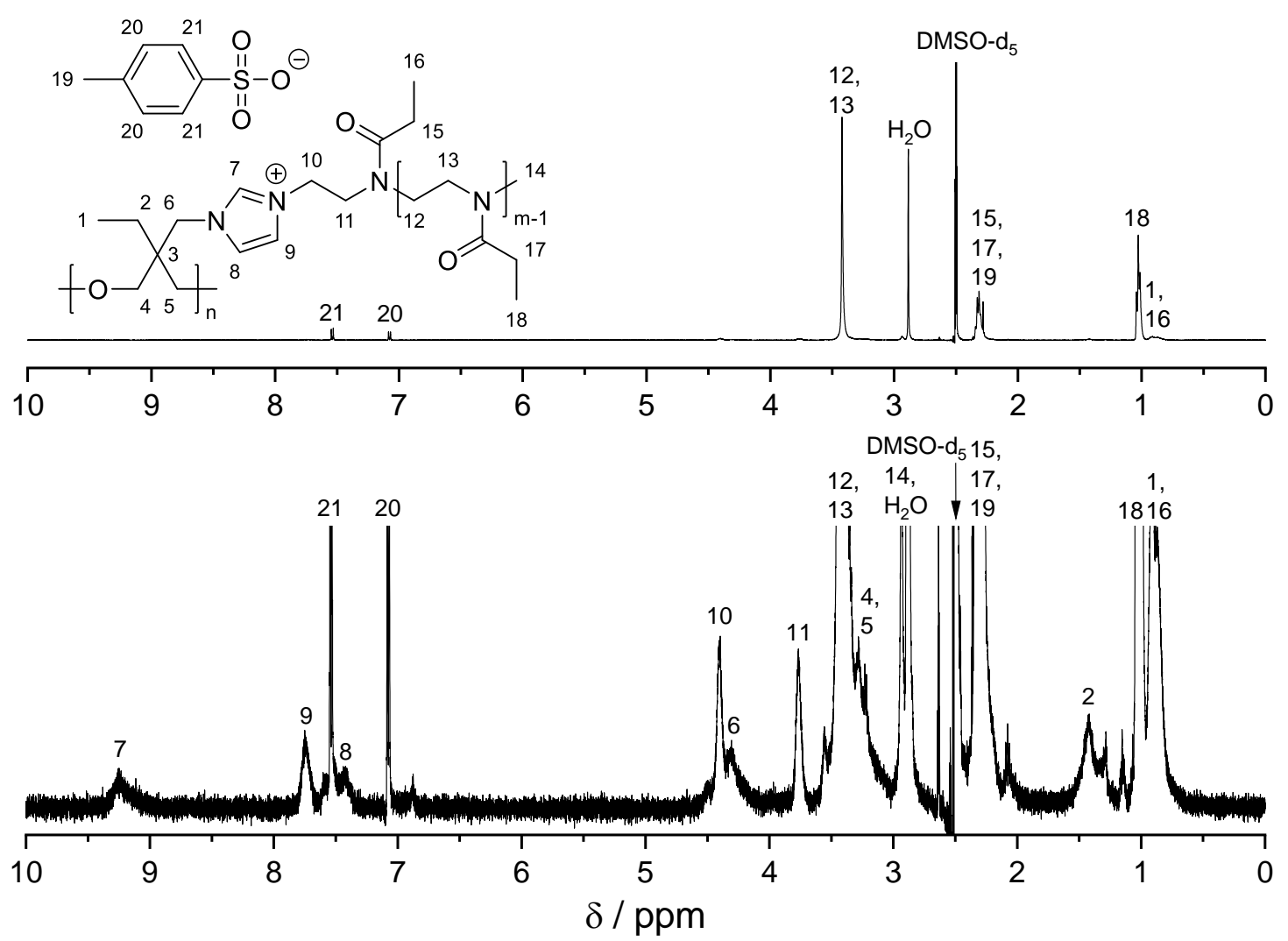

Figure S9: ${ }^{1} \mathrm{H}$ NMR spectrum of PEHO-IL-PEtOx 20 with magnification below. 

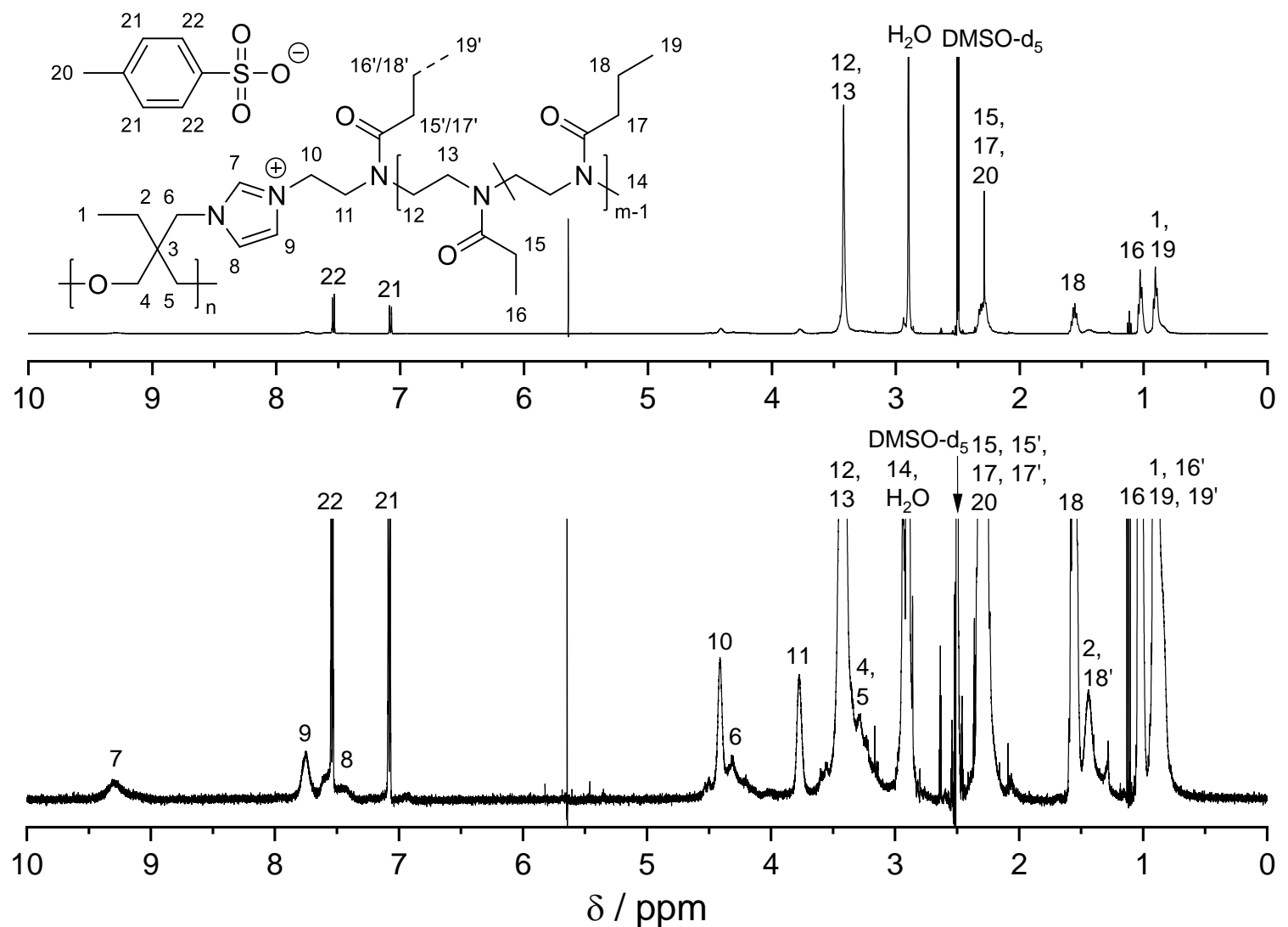

Figure S10: ${ }^{1} \mathrm{H}$ NMR spectrum of PEHO-IL-P(EtOx/nPrOx $)_{10}$ with magnification below.

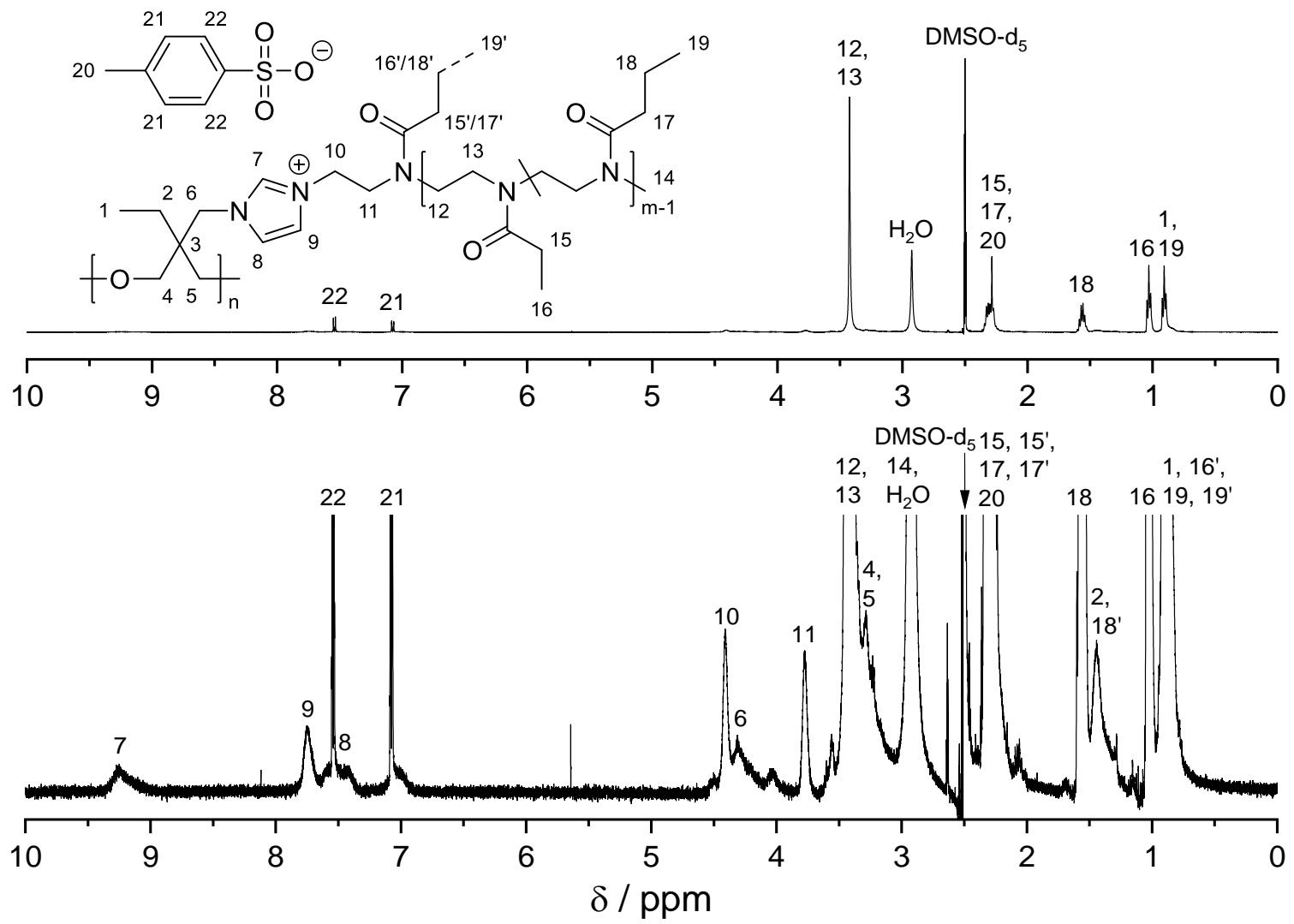

Figure S11: ${ }^{1} \mathrm{H}$ NMR spectrum of PEHO-IL-P(EtOx/nPrOx $)_{20}$ with magnification below. 


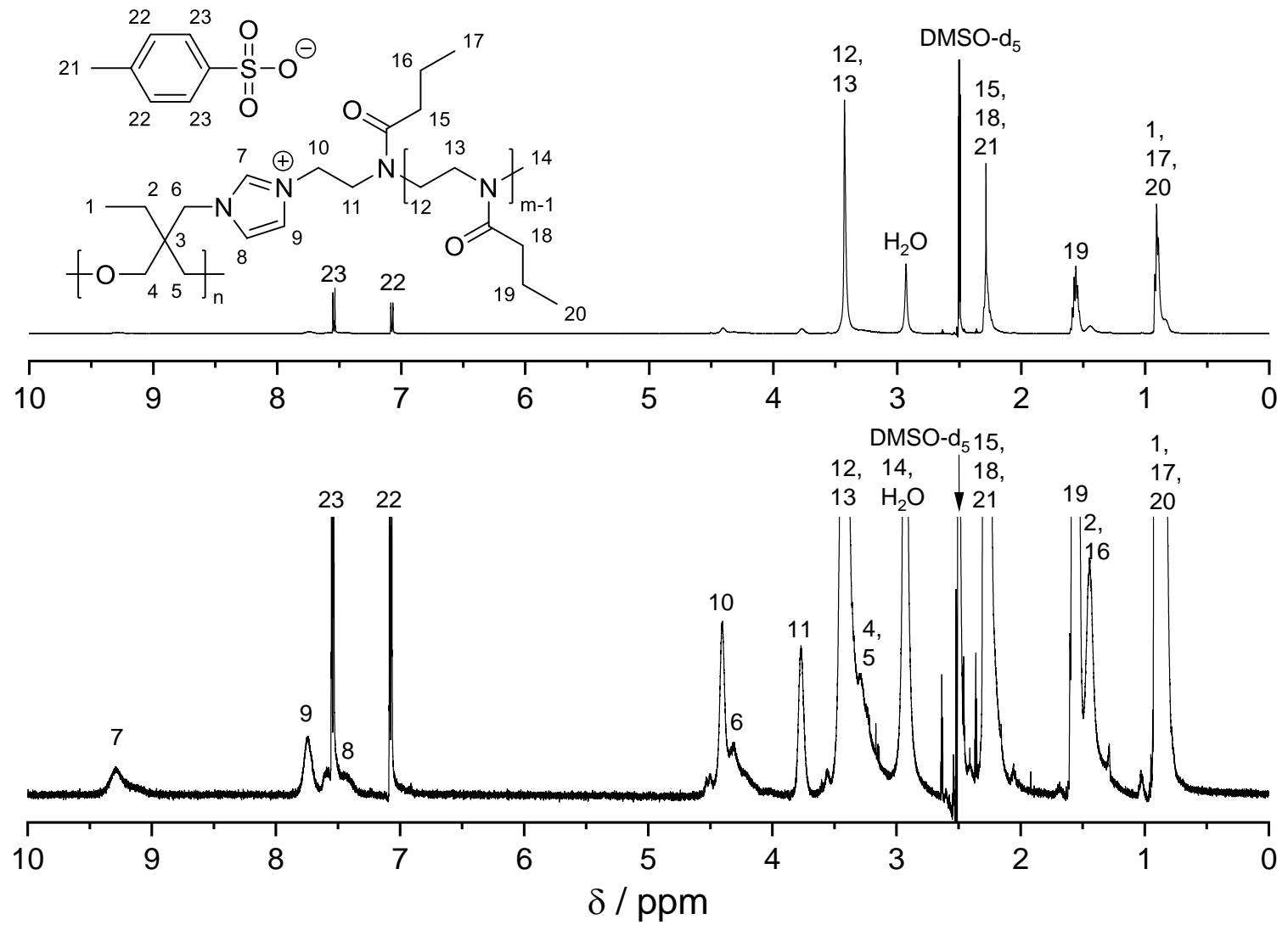

Figure S12: ${ }^{1} \mathrm{H}$ NMR spectrum of PEHO-IL-PnPrOx 10 with magnification below.

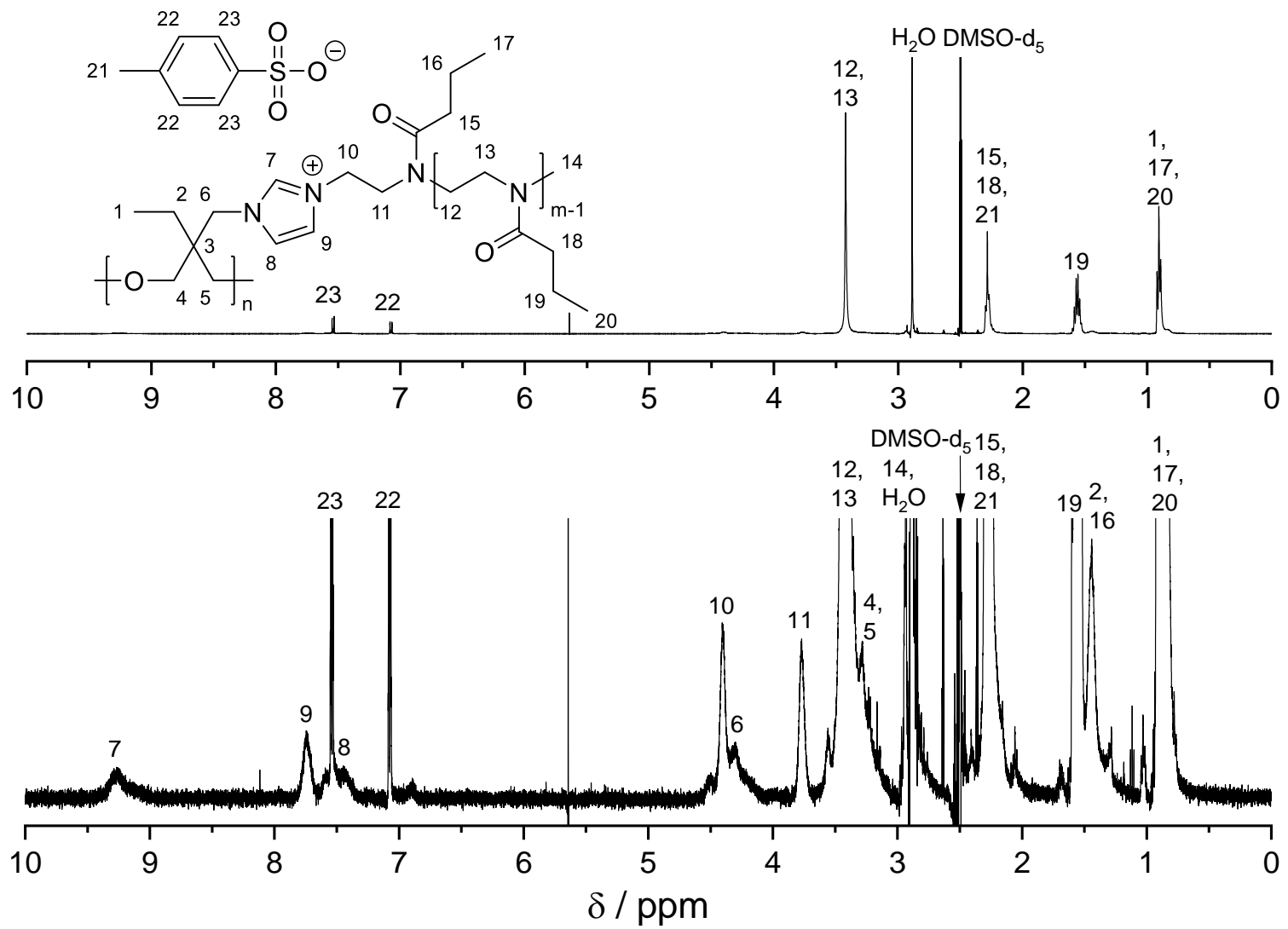

Figure S13: ${ }^{1} \mathrm{H}$ NMR spectrum of PEHO-IL-PnPrOx 20 with magnification below. 


\subsection{SEC curves}

- precipitated in $\mathrm{Et}_{2} \mathrm{O}$ - precipitated in $\mathrm{Et}_{2} \mathrm{O}+$ purified by TIPS $\quad$ discarded after TIPS
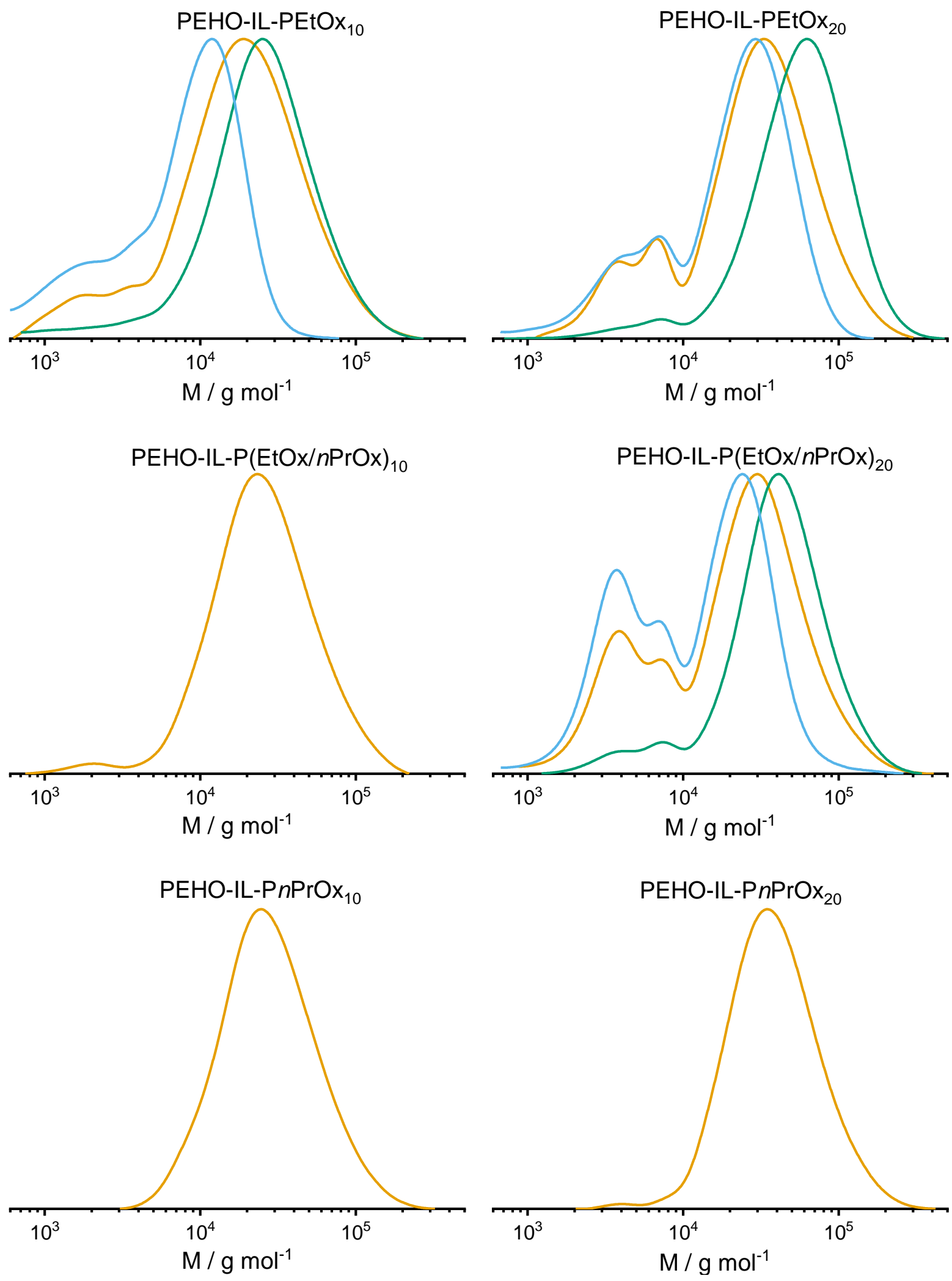

Figure S14: SEC curves of the hyperPILs after the respective purification steps (TIPS = temperature-induced phase separation, for details see experimental section). 


\subsection{Listings of low-molecular-weight fractions, yields and purities}

Table S2: Percentage of low-molecular-weight impurities in PEHO-IL-POx $x_{10}$ samples after precipitation in $\mathrm{Et}_{2} \mathrm{O}$ and, if applied, subsequent TIPS.

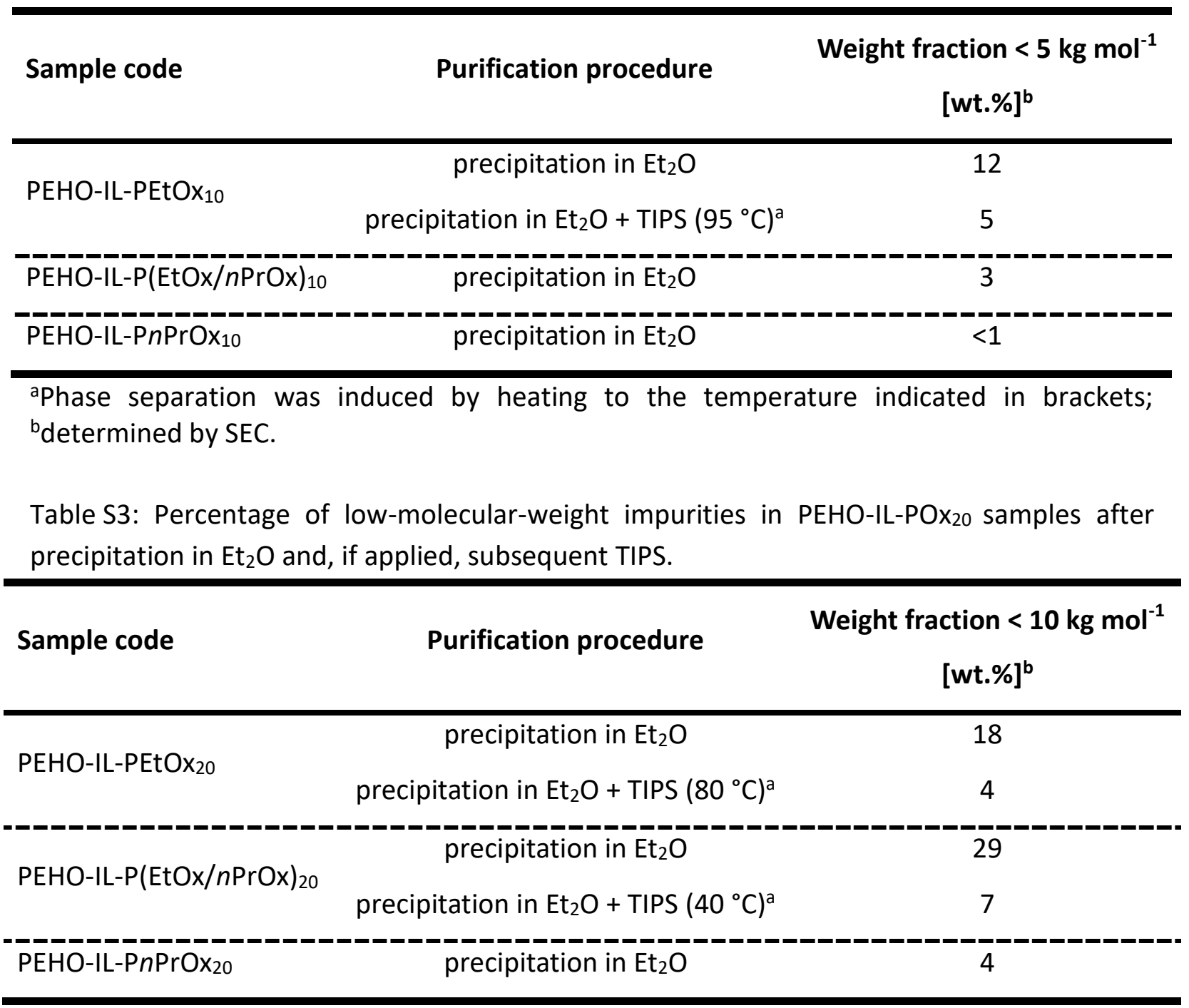

aPhase separation was induced by heating to the temperature indicated in brackets;

${ }^{\mathrm{b}}$ determined by SEC. 
Table S4: Yields and purities of the hyperPILs after precipitation in $\mathrm{Et}_{2} \mathrm{O}$ and, if applied, subsequent TIPS.

\begin{tabular}{|c|c|c|c|}
\hline Sample code & Purification procedure $^{a}$ & $\begin{array}{l}\text { Purity } \\
\text { [wt.\%] }\end{array}$ & $\begin{array}{l}\text { Yield } \\
\text { [g } \mid \%]\end{array}$ \\
\hline PEHO-IL-PEtO $x_{10}$ & precipitation in $\mathrm{Et}_{2} \mathrm{O}+\operatorname{TIPS}\left(95^{\circ} \mathrm{C}\right)$ & 95 & $0.62^{c} \mid 56$ \\
\hline PEHO-IL-PEtOx 20 & precipitation in $\mathrm{Et}_{2} \mathrm{O}+\operatorname{TIPS}\left(80^{\circ} \mathrm{C}\right)$ & 96 & $0.27^{c} \mid 24$ \\
\hline PEHO-IL-P(EtOx/nPrOx $)_{10}$ & precipitation in $\mathrm{Et}_{2} \mathrm{O}$ & 97 & $1.33 \mid 85$ \\
\hline PEHO-IL-P(EtOx/nPrOx) 20 & precipitation in $\mathrm{Et}_{2} \mathrm{O}+\operatorname{TIPS}\left(40^{\circ} \mathrm{C}\right)$ & 93 & $0.37^{c} \mid 36$ \\
\hline PEHO-IL-PnPrOx 10 & precipitation in $\mathrm{Et}_{2} \mathrm{O}$ & 99 & $1.98 \mid 74$ \\
\hline PEHO-IL-PnPrOx 20 & precipitation in $\mathrm{Et}_{2} \mathrm{O}$ & 96 & $2.45 \mid 80$ \\
\hline
\end{tabular}

aPhase separation was induced by heating to the temperature indicated in brackets; ${ }^{b}$ purity is defined as the percentage by weight of the fraction with molar masses larger than $5 \mathrm{~kg} \mathrm{~mol}^{-1}$ (for PEHO-IL-POx 10 samples) or $10 \mathrm{~kg} \mathrm{~mol}^{-1}$ (for PEHO-IL-POx 20 samples), as determined by SEC analysis; 'for the purification by TIPS only a part of the crude product obtained after $\mathrm{Et}_{2} \mathrm{O}$ precipitation was used (1.0 g for each sample). 


\subsection{Procedure for calculating $M_{n}$ from the ${ }^{1} \mathrm{H}$ NMR spectra}

In the ${ }^{1} \mathrm{H}$ NMR spectra of the hyperPILs, the signals of the imidazole groups either completely disappeared or are at least of such low intensity that a meaningful quantitative analysis by integration is not possible. Therefore, in good approximation, a quantitative alkylation of the imidazole groups with polyoxazoline chains can be assumed for estimation of the molecular weights. Accordingly, the integral of a proton of the imidazolium rings and the polyoxazoline chains corresponds to 0.82 times $(D F(P E H O-I m)=0.82)$ the integral of a proton of a repeat unit of the PEHO core. To normalize the integrals, the signal of the $\mathrm{CH}_{2}$ group $\left(\mathrm{H}^{11}\right)$ of the polyoxazoline repeat unit directly bonded to the imidazolium cation was selected and its integral was set to 1.64 .

The DP of the attached polyoxazoline chains can now be determined from the signal of the polyoxazoline backbone or from the signals of the $\mathrm{CH}_{2}$ - und $\mathrm{CH}_{3}$ - units of the side chains. The expected contributions of any overlapping signals are subtracted from the integral value of the respective signal. The remaining integral value is then divided by the product of DF (PEHO-Im) and the number of protons per repeat unit that contribute to the signal, which gives DP of the polyoxazoline chains. In case of using the polyoxazoline backbone signal, the obtained value must be increased by 1 , since the signals of the repeat unit directly bonded to the imidazolium cation do not overlap with those of the remaining repeat units. If several signals are suitable for determining DP, the arithmetic mean of the calculated DP values based on different signals is given.

To calculate the $M_{n}$ of the hyperPILs, DP of the polyoxazoline chains is first multiplied with the molar mass of the respective monomer and the molar mass of the initiator (MeOTs) is added to obtain the $M_{\mathrm{n}}$ of a single polyoxazoline chain. This value is multiplied with the number of imidazole units per PEHO-Im molecule, which is calculated by multiplying DF (PEHO-Im) (0.82) with DP of the PEHO core (15). Finally, to the $M_{n}$ of the polyoxazoline shell obtained in this way, the $M_{\mathrm{n}}$ of PEHO-Im ( $2500 \mathrm{~g} \mathrm{~mol}^{-1}$ ) is added to obtain $M_{\mathrm{n}}$ of the hyperPILs.

This calculation is shown below by the example of PEHO-IL-PnPrOx $10 . \mathrm{DP}_{1}, \mathrm{DP}_{2}$ etc. denote the degrees of polymerization determined from different signals. From these values, the arithmetic mean is formed. 


$$
\begin{gathered}
D P_{1}=\frac{I\left(H^{1}+H^{17}+H^{20}\right)-3}{3 * D F}=11.6 \\
D P_{2}=\frac{I\left(H^{2}+H^{16}+H^{19}\right)-2}{2 * D F}=11.7 \\
D P_{3}=\frac{I\left(H^{15}+H^{18}+H^{21}\right)-\frac{3}{2} * I\left(H^{22}\right)}{2 * D F}=11.3 \\
D P_{4}=\frac{I\left(H^{4}+H^{5}+H^{12}+H^{13}\right)-4}{4 * D F}+1=11.2 \\
\overline{D P}=\frac{D P_{1}+D P_{2}+D P_{3}+D P_{4}}{4}=11.4
\end{gathered}
$$

$M_{n}\left(\right.$ PEHO-IL-PnPrOx $\left.x_{10}\right)$

$$
\begin{aligned}
& =(((\overline{D P}(\text { PnPrOx }) * M(\text { nPrOx }))+M(\text { MeOTs })) \\
& * \text { DF }(\text { PEHO-Im }) * D P(\text { PEHO-Im }))+M_{n}(\text { PEHO-Im }) \\
& =\left(\left(\left(11.4 * 113 \frac{\mathrm{g}}{\mathrm{mol}}\right)+186 \frac{\mathrm{g}}{\mathrm{mol}}\right) * 0.82 * 15\right)+2500 \frac{\mathrm{g}}{\mathrm{mol}} \\
& =21000 \frac{\mathrm{g}}{\mathrm{mol}}
\end{aligned}
$$




\subsection{Thermal characterization by TGA and DSC}
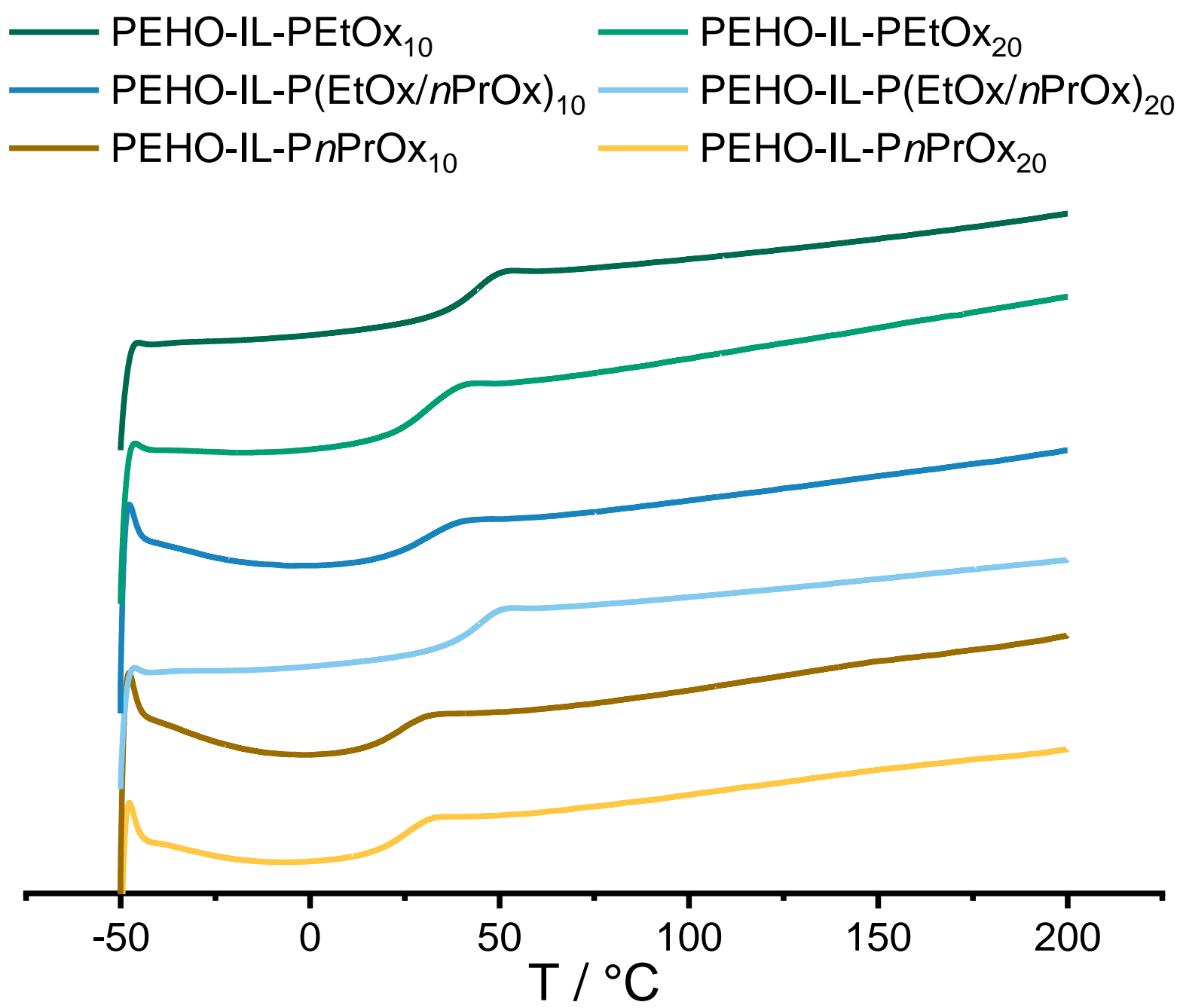

Figure S15: DSC thermograms ( $2^{\text {nd }}$ heating curve) of the hyperPILs.
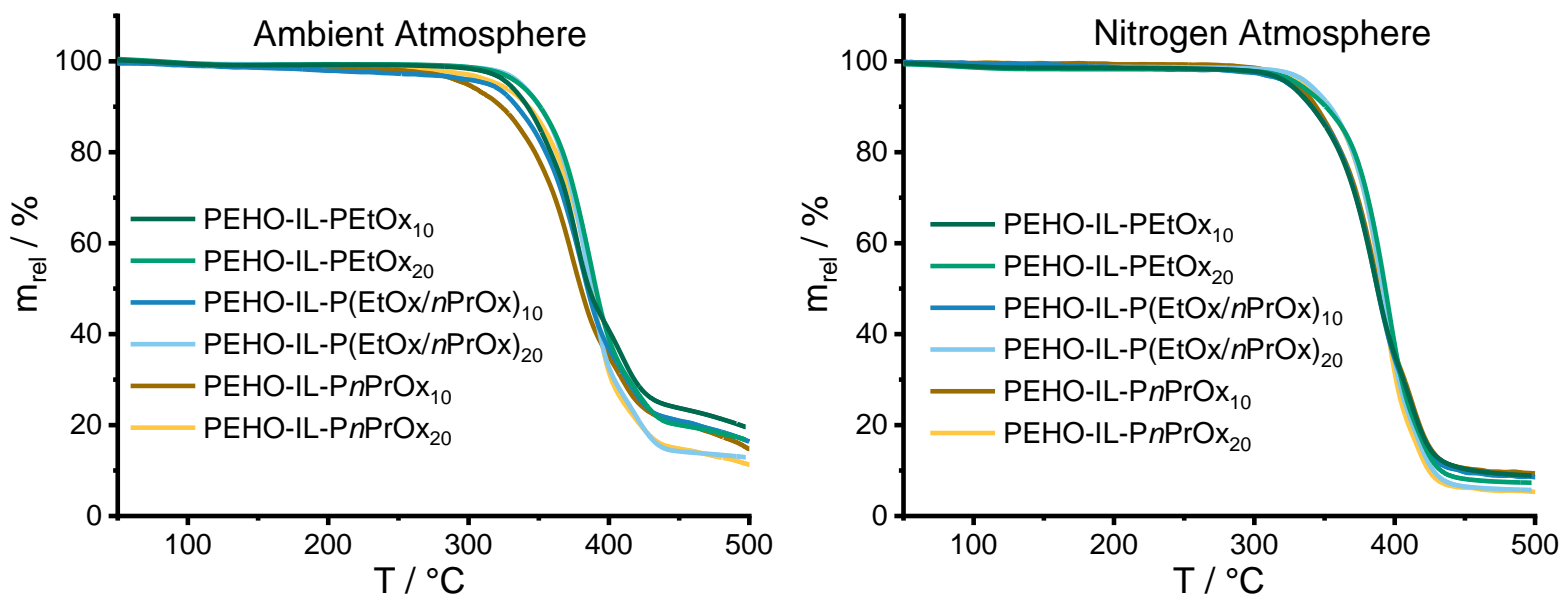

Figure S16: TGA curves of the hyperPILs under air (left) and nitrogen atmosphere (right). 


\subsection{Transmission-temperature curves}
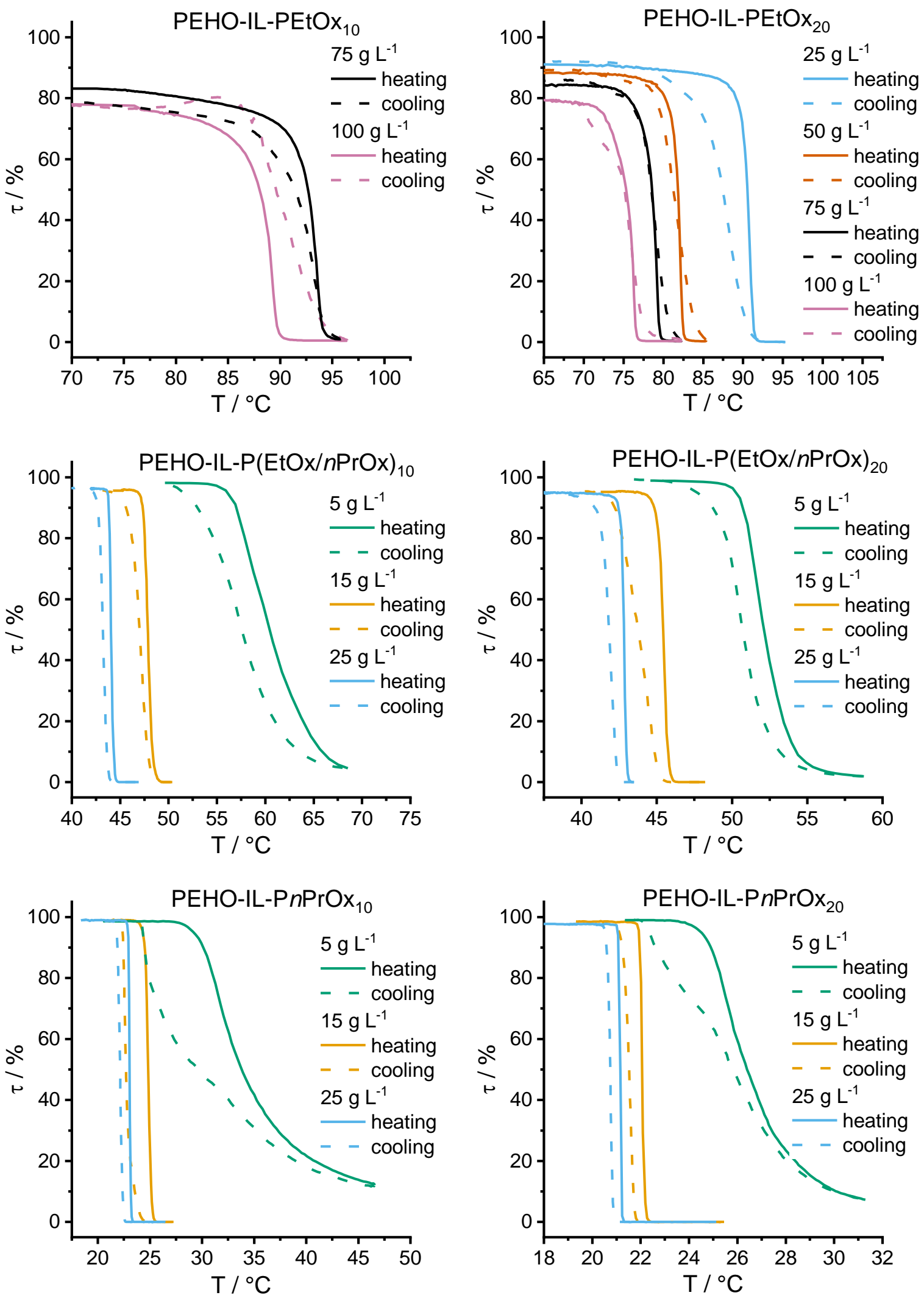

Figure S17: Transmission-temperature curves of aqueous hyperPIL solutions (heating/cooling rate $\left.=1 \mathrm{~K} \mathrm{~min}^{-1}\right)$. 


\subsection{Listing of cloud points ( $\left.T_{\mathrm{CP}}\right)$}

Table S5: $T_{\mathrm{CP}}$ values of hyperPILs at various concentrations.

\begin{tabular}{|c|c|c|c|c|c|c|}
\hline \multirow{2}{*}{ Sample code } & \multicolumn{6}{|c|}{$T_{\mathrm{CP}}\left[{ }^{\circ} \mathrm{C}\right]^{\mathrm{a}}$} \\
\hline & $5 \mathrm{~g} \mathrm{~L}^{-1}$ & $15 \mathrm{~g} \mathrm{~L}^{-1}$ & $25 \mathrm{~g} \mathrm{~L}^{-1}$ & $50 \mathrm{~g} \mathrm{~L}^{-1}$ & $75 \mathrm{~g} \mathrm{~L}^{-1}$ & $100 \mathrm{~g} \mathrm{~L}^{-1}$ \\
\hline PEHO-IL-PEtOx 10 & - & - & - & - & 93 & 89 \\
\hline PEHO-IL-PEtOx 20 & - & - & 91 & 82 & 79 & 76 \\
\hline PEHO-IL-P(EtOx $/ n$ PrOx $)_{10}$ & 60 & 48 & 44 & nd & nd & nd \\
\hline PEHO-IL-P(EtOx $/ n$ PrOx $)_{20}$ & 52 & 45 & 43 & nd & nd & nd \\
\hline PEHO-IL-PnPrOx 10 & 32 & 25 & 23 & nd & nd & nd \\
\hline PEHO-IL-PnPrOx 20 & 26 & 22 & 21 & nd & nd & nd \\
\hline
\end{tabular}

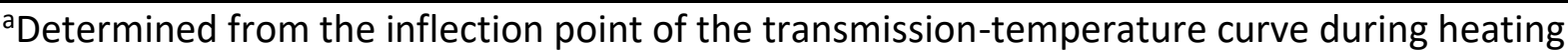
$\left(\lambda=500 \mathrm{~nm}\right.$; heating rate $\left.=1 \mathrm{~K} \mathrm{~min}^{-1}\right) ;-=$ no clouding detected, $\mathrm{nd}=$ not determined.

\subsection{TEM images}
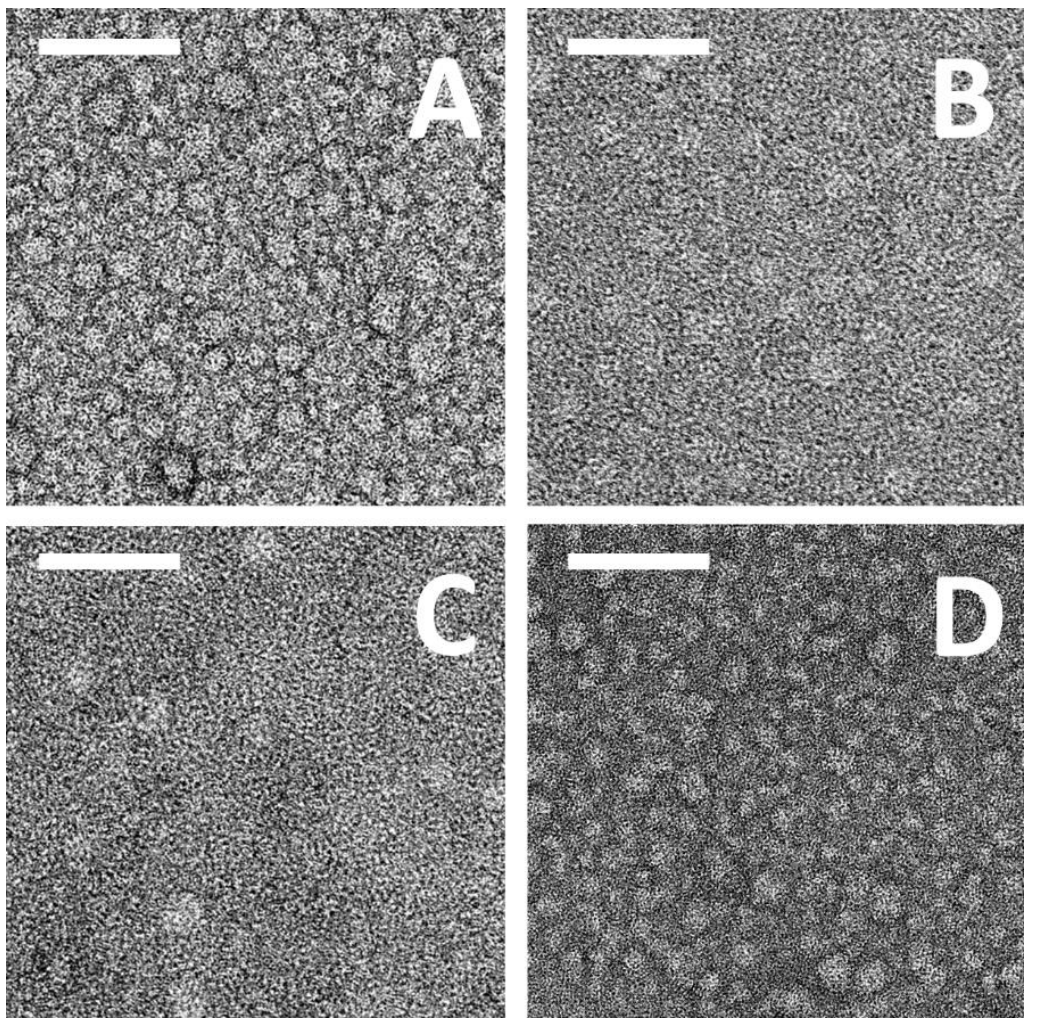

Figure S18: TEM images of PEHO-IL-PEtOx 10 (A), PEHO-IL-PEtOx 20 (B), PEHO-IL-PnPrOx 10 (C), and PEHO-IL-PnPrOx 20 (D); scale bar $=100 \mathrm{~nm}$. 


\subsection{Chemical structures of the molecules used for the transport experiments}

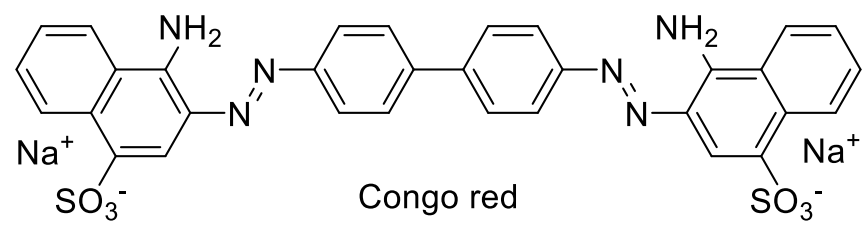

Methylene blue<smiles>CN(C)c1ccc2nc3ccc(=[N+](C)C)cc-3sc2c1</smiles><smiles>Cc1ccccc1/N=N/c1ccc(/N=N/c2c(O)ccc3ccccc23)c(C)c1</smiles>

Figure S19: Chemical structures of Congo red, Methylene blue, and Sudan IV.

phosphine ligand

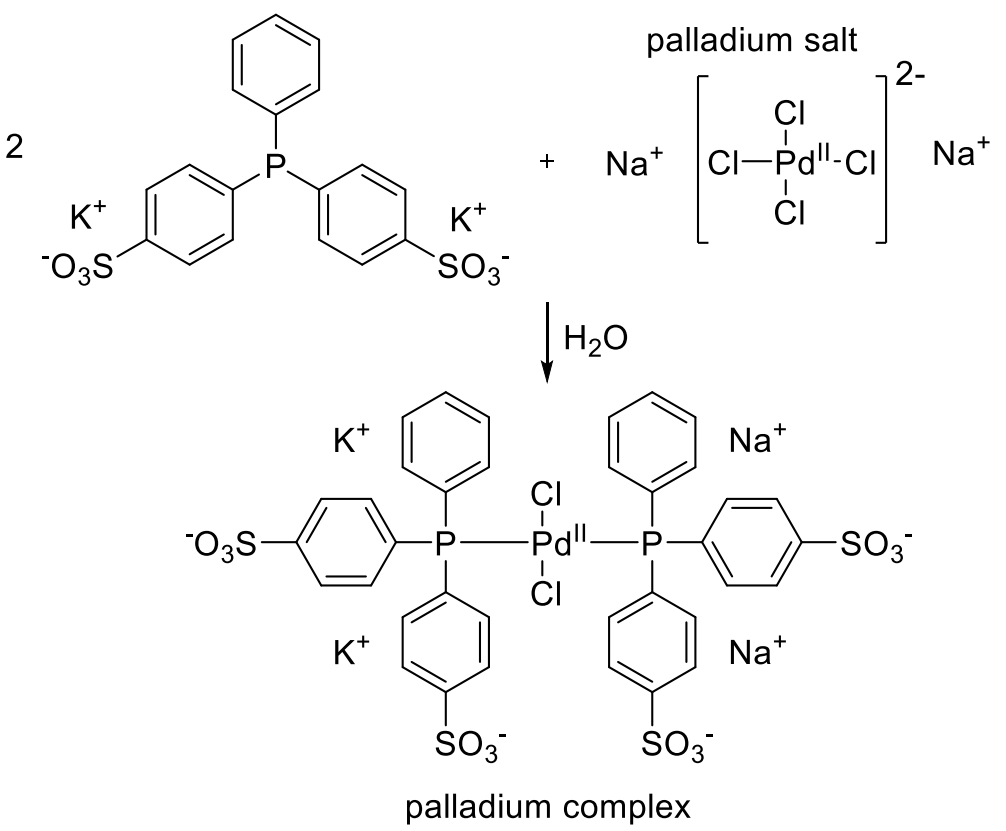

Figure S20: Proposed complex forming reaction and structure of the Pd-phosphine complex.

\section{References}

(1) Bednarek, M.; Biedron, T.; Helinski, J.; Kaluzynski, K.; Kubisa, P.; Penczek, S. Branched polyether with multiple primary hydroxyl groups: polymerization of 3-ethyl-3hydroxymethyloxetane. Macromol. Rapid Commun. 1999, 20, 369-372.

(2) Hölter, D.; Burgath, F.; Frey, H. Degree of branching in hyperbranched polymers. Acta Polym. 1997, 48, 30-35. 